\title{
ARTICLE
}

Received 10 Nov 2015 | Accepted 1 Dec 2015 | Published 12 Jan 2016

DOI: $10.1038 /$ ncomms10332

OPEN

\section{Impairment of PARK14-dependent $\mathrm{Ca}^{2}+$ signalling is a novel determinant of Parkinson's disease}

Qingde Zhou ${ }^{1, \star}$, Allen Yen ${ }^{1, \star}$, Grzegorz Rymarczyk ${ }^{1}$, Hirohide Asai ${ }^{2}$, Chelsea Trengrove ${ }^{2}$, Nadine Aziz ${ }^{1}$ Michael T. Kirber ${ }^{1}$, Gustavo Mostoslavsky ${ }^{3}$, Tsuneya Ikezu², Benjamin Wolozin ${ }^{2}$ \& Victoria M. Bolotina ${ }^{1}$

The etiology of idiopathic Parkinson's disease (idPD) remains enigmatic despite recent successes in identification of genes (PARKs) that underlie familial PD. To find new keys to this incurable neurodegenerative disorder we focused on the poorly understood PARK14 disease locus (Pla2g6 gene) and the store-operated $\mathrm{Ca}^{2+}$ signalling pathway. Analysis of the cells from idPD patients reveals a significant deficiency in store-operated PLA2g6-dependent $\mathrm{Ca}^{2+}$ signalling, which we can mimic in a novel B6.Cg-Pla2g6 ${ }^{4 \mathrm{Ex} 2-\mathrm{VB}}\left(\mathrm{PLA} 2 \mathrm{~g} 6 \mathrm{ex} 2^{\mathrm{KO}}\right)$ mouse model. Here we demonstrate that genetic or molecular impairment of PLA2g6-dependent $\mathrm{Ca}^{2}+$ signalling is a trigger for autophagic dysfunction, progressive loss of dopaminergic (DA) neurons in substantia nigra pars compacta and age-dependent L-DOPA-sensitive motor dysfunction. Discovery of this previously unknown sequence of pathological events, its association with idPD and our ability to mimic this pathology in a novel genetic mouse model opens new opportunities for finding a cure for this devastating neurodegenerative disease.

\footnotetext{
${ }^{1}$ Department of Medicine, Boston University School of Medicine, Boston, Massachusetts 02118, USA. ${ }^{2}$ Department of Pharmacology and Experimental Therapeutics, Boston University School of Medicine, Boston, Massachusetts 02118, USA. ${ }^{3}$ Center for Regenerative Medicine, Boston University School of Medicine, Boston, Massachusetts 02118, USA. * These authors contributed equally to this work. Correspondence and requests for materials should be addressed to V.M.B. (email: bolotina@bu.edu).
} 
G enetic mutations in specific genes (PARKs) are thought to be the primary risk factors for familial Parkinson's disease (fPD), which comprises about $10 \%$ of all Parkinson's disease (PD) cases. Several PARKs have been linked to autophagic dysfunction, mitochondrial dysfunction, $\alpha$-synuclein aggregation and other cellular defects, which are now viewed as hallmarks of human $\mathrm{PD}^{1-4}$. However, the vast majority of $\mathrm{PD}$ cases are idiopathic, occurring on ageing with no distinct triggers or clear underlying mechanisms. The aetiology of idiopathic PD (idPD) remains obscure, suggesting that not all pathological mechanisms underlying this disease may have been accounted for.

To find new triggers and signalling pathways that can lead to age-dependent idPD, we focused on the store-operated $\mathrm{Ca}^{2+}$ signalling and the poorly understood disease locus PARK14, which was identified as the Pla2g6 gene ${ }^{5}$ encoding $\mathrm{Ca}^{2+}$. independent phospholipase A2 group 6 (PLA2g6 or iPLA $_{2} \beta$ ). Distinct mutations in Pla2g6 gene (PARK14) were found to be associated with familial Parkinsonism ${ }^{6-11}$, although the underlying mechanism and the role of PLA2g6 in idPD remain unclear. The PLA2g6 is a multifaceted enzyme that is best known for its catalytic function, which was linked to phospholipid remodelling in cells (for review see ref. 12). The loss of the catalytic activity of PLA2g6 was associated with infantile neuroaxonal dystrophy (INAD), and results in early death in humans and in mouse models ${ }^{13-19}$. In contrast to INAD mutations, PD-associated mutations in PLA2g6 were reported not to affect its catalytic activity ${ }^{20}$. The question remains open as to which cellular function of PLA2g6 could be involved in human $\mathrm{PD}$, and may be responsible for a PD-like phenotype that would appear later with ageing.

Earlier, we discovered ${ }^{21-26}$ and others confirmed ${ }^{27-31}$ that PLA2g6 plays an important role in the activation of endogenous store-operated $\mathrm{Ca}^{2+}$ entry (SOCE). Notably, besides Orail (store-operated plasma membrane $\mathrm{Ca}^{2+}$ channel) and STIM1 $\left(\mathrm{Ca}^{2}+\right.$ sensor in endoplasmic reticulum (ER)), PLA2g6 was identified in an RNAi screen as one of the essential components of endogenous SOCE (supplementary material in ref. 32). It is well established that SOCE is activated on depletion of ER $\mathrm{Ca}^{2+}$ stores (for review see refs 33-35), and is crucial for their timely refilling in a wide variety of cell types. However, the role of store-operated $\mathrm{Ca}^{2}+$ signalling in dopaminergic (DA) neurons and PD remains largely unknown.

This study was designed to explore the origins of human age-dependent PD from the new perspective of PARK14 and the store-operated $\mathrm{Ca}^{2}+$ signalling. We tested a novel idea that impairment of the store-dependent $\mathrm{Ca}^{2+}$ signalling function of PARK14 could be a previously unknown determinant of idPD and PARK14 form of fPD. Using a wide array of genetic, molecular, cellular, imaging, behaviour and other approaches for in vivo and in vitro studies in human cells and new mouse model, we discovered a major source for cellular vulnerability that can be associated with premature death of DA neurons and age-dependent PD.

\section{Results}

SOCE and PLA2g6 are impaired in idPD and $\mathrm{fPD}{ }^{\mathrm{R} 747 \mathrm{~W}}$ patients. One of the requirements for $\mathrm{Ca}^{2+}$ signalling studies is the live-cell preparation. Because live DA neurons cannot be extracted from the midbrain of PD patients, human primary skin fibroblasts (hPSFs) provide an alternative cellular model representing PD and control patients, which proved to be instrumental for the studies of human neurodegeneration. To determine if idPD may be associated with changes in store-operated $\mathrm{Ca}^{2+}$ signalling, primary skin fibroblasts from control donors and idPD patients were obtained from the NINDS Cell Line Repository (http://ccr.coriell.org/ninds; Supplementary Table 1), and used in our studies. The idPD group was represented by aged ( $62 \pm 5$ years old) male Caucasian idPD patients with no documented mutations in the PARK genes and no family history of PD. The group of neurological control donors was represented by aged (68 \pm 8 years old) Caucasian males with no symptoms or family history of PD.

Analysis of live primary skin fibroblasts (P3-5) from idPD and control donors revealed a significant deficit in endogenous SOCE in the cells from all idPD patients tested (Fig. 1a,c and Supplementary Fig. 1): on average, there was more than $40 \%$ reduction in thapsigargin (TG)-induced $\mathrm{Ca}^{2}+$ influx in idPD patients compared with the control group. SOCE in both groups was highly sensitive to diethylstilbestrol (inhibitor of PLA2g6dependent Orail-mediated $\mathrm{SOCE}^{36,37}$ ): $10 \mu \mathrm{M}$ of diethylstilbestrol produced $80 \pm 1 \%$ and $82 \pm 4 \%$ inhibition of TG-induced $\mathrm{Ca}^{2+}$ influx in control and idPD groups, respectively.

Importantly, we found that the cells from a patient with fPD associated with R747W mutation in PARK14/PLA2g6 $\left(\mathrm{fPD}^{\mathrm{R} 747 \mathrm{~W}}\right.$ ) have a similar deficit in SOCE (Fig. 1b,c and Supplementary Fig. 1).

To understand which PLA2g6 function may be associated with human PD, catalytic activity and store-dependent activation of PLA2g6 was analysed and compared in the cells from control, $\mathrm{idPD}$ and $\mathrm{fPD}^{\mathrm{R} 747 \mathrm{~W}}$ patients. We found that activation of PLA2g6 by TG-induced $\mathrm{Ca}^{2+}$ store depletion ${ }^{26}$ seen in control donors is significantly impaired in the cells from idPD and $\mathrm{fPD}^{\mathrm{R} 747 \mathrm{~W}}$ patients, while the catalytic activity of PLA2g6 is the same (Fig. 1d,e and Supplementary Fig. 2a,b). The specificity of PLA2g6 activity in both cases was confirmed by its inhibition with S-BEL, a chiral-specific suicidal substrate that discriminates PLA2g6 from all other phospholipases ${ }^{38}$.

Notably, the analysis of the expression of several major components of SOCE revealed that SOCE deficiency in the cells from idPD patients was not associated with reduction in Orai1, TRPC1, STIM1 or STIM2 expression: Fig. If and Supplementary Figs 3 and 4 demonstrate no difference in mRNA levels for these genes in control and PD patients. In contrast, we found that the expression of PLA2g6(L) (a specific plasma membrane-associated splice variant of PLA2g6 (ref. 39)) is significantly reduced in idPD patients (Fig. If and Supplementary Fig. 3b), while expression of the PLA2g6(S) splice variant (which lacks exon $8 \mathrm{~b}$ and encodes a cytosolic enzyme that was shown to be involved in lipid remodelling ${ }^{12}$ ) is the same, as in control donors.

Thus, primary skin fibroblasts from idPD and $\mathrm{PPD}^{\mathrm{R} 747 \mathrm{~W}}$ patients share a striking deficiency in store-dependent activation of PLA2g6, as well as significant impairment in endogenous SOCE.

PLA2g6 ex2 ${ }^{\mathrm{KO}}$ mice mimic SOCE deficiency in idPD patients. To determine if and how the defects in the store-dependent activation of PLA2g6 and impaired SOCE could be translated into the age-dependent $\mathrm{PD}$, we sought a mouse model with targeted impairment of these specific cellular functions. Currently existing transgenic PLA2g6 mouse models ${ }^{13-18}$ appear to be unsuitable for PD studies, as they have impaired catalytic activity of PLA2g6, which we found to be unaffected in idPD patients. Moreover, PD pathology develops later in life, while the loss of catalytic activity of PLA2g6 is associated with INAD and early death in mice and humans.

To create a new model suitable for PD studies, we targeted PLA2g6 in a different manner. We hypothesized that genetic deletion of the $\mathrm{N}$ terminus of PLA2g6 (Fig. 2a) should not affect 
a

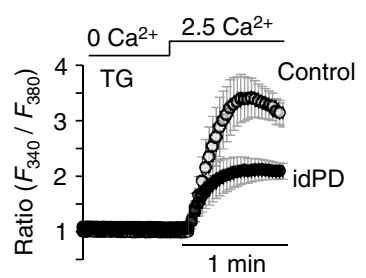

b

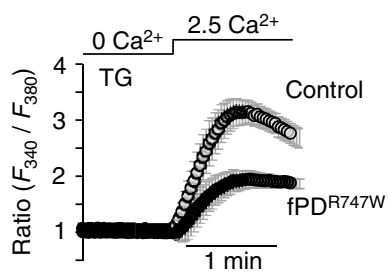

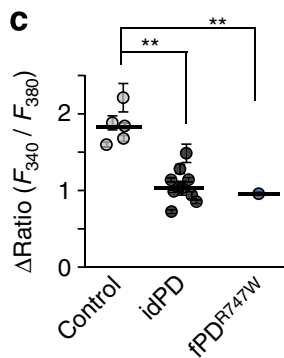

d

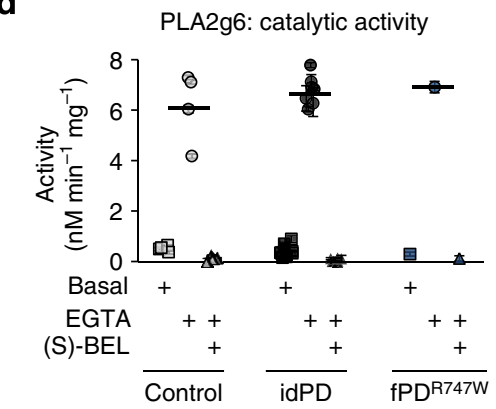

e PLA2g6: store-dependent activation

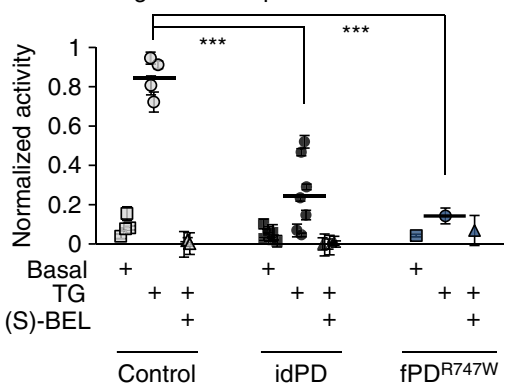

f
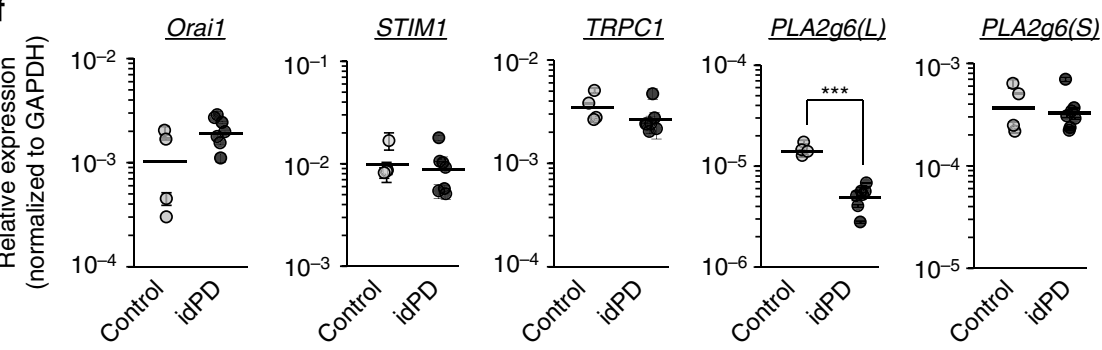

Figure 1 | Deficiency in PARK14 and store-operated $\mathrm{Ca}^{2}+$ signalling in human primary skin fibroblasts from patients with idPD and with familial PLA2g6 ${ }^{\mathbf{R 7 4 7 W}}$ mutation (fPD ${ }^{\mathbf{R 7 4 7 W}}$ ). (a,b) Representative traces show SOCE on Ca ${ }^{2+}$ addition in TG-pretreated ( $5 \mu \mathrm{M}$ for 20 min) fibroblasts from control, idPD patients (in $\mathbf{a}$ ) and $\mathrm{fPD} \mathrm{R}^{\mathrm{R} 747 \mathrm{~W}}$ patient (in b): each trace show $\mathrm{Ca}^{2+}$ influx (average Ratio $\left(F_{340} / F_{380}\right) \pm$ s.d.) in a group of $10-20$ individual cells measured simultaneously; full traces are shown in Supplementary Fig. 1. (c) Comparative analysis of SOCE in fibroblasts from control idPD and fPD R747W patients. Data for each patient show the average \pm s.e. from at least three independent experiments, with up to 120 cells analysed for each patient (see Supplementary Fig. 1 for details). (d) Catalytic PLA2g6 activity in homogenates of fibroblasts from control, idPD and fPDR747W patients: summary results show average activity ( \pm s.e.) from three repetitions under basal conditions, after activation in the presence of $10 \mathrm{mM}$ EGTA and after inhibition with $25 \mu \mathrm{M}$ of (S)-BEL (see Supplementary Fig. 2a for details). (e) Activation of PLA2g6 by TG-induced depletion of $\mathrm{Ca}^{2+}$ stores in intact fibroblasts from control, idPD and $\mathrm{fPD}^{\mathrm{R} 747 \mathrm{~W}}$ patients: summary results show average activity ( \pm s.e.) from three repetitions under basal conditions, after activation by $\mathrm{TG}(5 \mu \mathrm{M}$ for $10 \mathrm{~min}$ ) and after inhibition with (S)-BEL (see Supplementary Fig. 2b for details). (f) Relative expression of Orai1, STIM1, TRPC1, PLA2g6(L) and PLA2g6(S) in fibroblasts from control and idPD patients: summary results of quantitative reverse transcription-PCR analysis normalized to GAPDH for each sample, with group averages shown by the horizontal line (see Supplementary Fig. 3 for details). ${ }^{\star \star} P<0.01$, ${ }^{\star \star \star} P<0.001$.

its catalytic activity (encoded by $\mathrm{S}^{519}$-containing catalytic domain in the $\mathrm{C}$ terminus), but may impair PLA2g6 activation by depleted stores, thus reproducing the specific deficiency that we found in idPD patients. To test this approach, a novel PLA2g6 ex $2^{\mathrm{KO}}$ mouse model was created $\left(\mathrm{B} 6 . \mathrm{Cg}-\mathrm{Pla} 2 \mathrm{~g} 6^{\Delta E x 2-V B} / \mathrm{J}\right)$, in which exon 2 of Pla2g6 gene was constitutively deleted (see Fig. 2a, Supplementary Figs 5-7 and Methods for details on generation and validation of this model). Deletion of the translation initiation $\mathrm{ATG}_{1}$ (coded by exon 2), did not affect expression of (L) and (S) splice variants of PLA2g6 (Supplementary Fig. 8), and did not lead to the loss of PLA2g6 protein: the presence of a cryptic $\mathrm{ATG}_{2}$ in exon 4 initiated translation and resulted in the expression of the truncated ex $2 \mathrm{KO}$ PLA2g6 protein that lacks the first 178 amino acids in the $\mathrm{N}$ terminus (Supplementary Fig. 9 and 10), while the rest of the molecule remained intact.

Analysis of the PLA2g6 activity in mouse embryonic fibroblasts (MEFs) from wild-type (WT) and ex $2^{\mathrm{KO}}$ animals revealed that genetic truncation of the $\mathrm{N}$ terminus did not affect its catalytic activity (Fig. 2b), but resulted in the loss of PLA2g6 activation by
TG-induced depletion of the stores (Fig. 2c). Thus, similar to the cells from idPD and $\mathrm{fPD}^{\mathrm{R} 747 \mathrm{~W}}$ patients, ex $2^{\mathrm{KO}}$ mice appear to have deficiency in the store-dependent activation of PLA2g6. It is important to emphasize that preserved catalytic activity of PLA2g6 clearly discriminates this new ex $2^{\mathrm{KO}}$ mouse model from other PLA2g6 models ${ }^{13-18}$, in which catalytic activity of this enzyme was genetically impaired.

To test if PLA2g6 ex2 $2^{\mathrm{KO}}$ mice could also mimic the defect of the store-operated $\mathrm{Ca}^{2}+$ signalling that we found in fibroblasts from idPD patients, endogenous SOCE was analysed and compared in MEFs from WT and ex $2^{\mathrm{KO}}$ animals. The results of these studies indicated that TG-induced SOCE is significantly impaired in MEFs from ex $2^{\mathrm{KO}}$ mice (Fig. 2d,e). Similarly, we found that TPEN (a low-affinity $\mathrm{Ca}^{2+}$ chelator that is known to mimic depletion of $\mathrm{Ca}^{2+}$ in ER stores ${ }^{40,41}$ ) activates PLA2g6-dependent (BEL-sensitive) SOCE in the cells from WT, but not from ex $2^{\mathrm{KO}}$ mice (Supplementary Fig. 11). Consistent with the physiological role of SOCE in refilling of ER $\mathrm{Ca}^{2+}$ stores, significant impairment of endogenous SOCE resulted in constitutive depletion of intracellular $\mathrm{Ca}^{2}+$ stores in $\mathrm{ex} 2^{\mathrm{KO}}$ cells, 
as indicated by more than $50 \%$ decrease in intracellular $\mathrm{Ca}^{2+}$ release caused by ionomycin (Fig. 2f,g). Passive $\mathrm{Ca}^{2}+$ release in response to TG application in the absence of extracellular $\mathrm{Ca}^{2+}$ (Fig. 2d) was also significantly decreased in $\mathrm{ex} 2^{\mathrm{KO}}$ cells (by $56 \pm 5 \%, P<0.01$ ).

Importantly, primary skin fibroblasts from idPD patients also appear to have significant reduction in ionomycin-induced $\mathrm{Ca}^{2+}$ release: the $\Delta$ Ratio $\left(F_{340} / F_{380}\right)$ was $5.9 \pm 0.5(n=7)$ in idPD and $8.5 \pm 0.9(n=4)$ in control patients, respectively $(P<0.05)$.

Therefore, PLA2g6 ex $2^{\mathrm{KO}}$ mice exhibit major functional deficiencies in store-operated PLA2g6-dependent $\mathrm{Ca}^{2+}$ signalling that mirrors the pathological cellular phenotype found in $\mathrm{idPD}$ and $\mathrm{fPD}^{\mathrm{R} 747 \mathrm{~W}}$ patients (Fig. 1): MEFs from ex $2^{\mathrm{KO}}$ mice (i) retain normal catalytic activity of PLA2g6 (Fig. 2b), (ii) have a major defect in PLA2g6 activation by depleted stores (Fig. 2c), (iii) have a significant impairment of endogenous SOCE and (iv) show a significant depletion of intracellular $\mathrm{Ca}^{2}+$ stores

a

PARK14: B6.129S-Pla2g6 $6^{4 E \times 2-V B} / J$ model

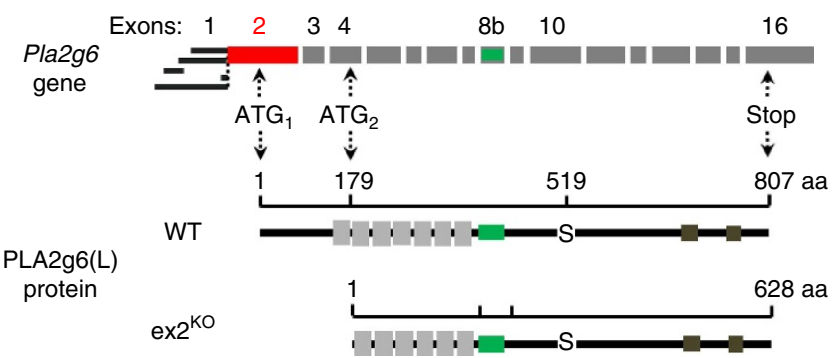

b

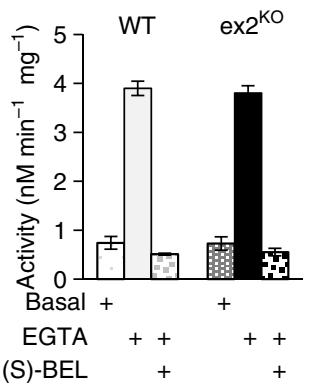

C

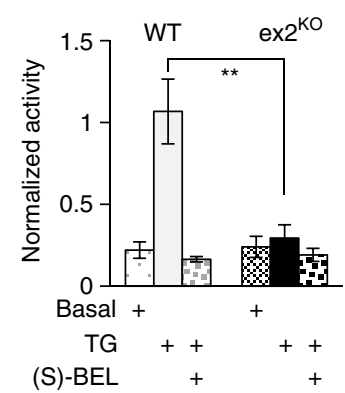

d

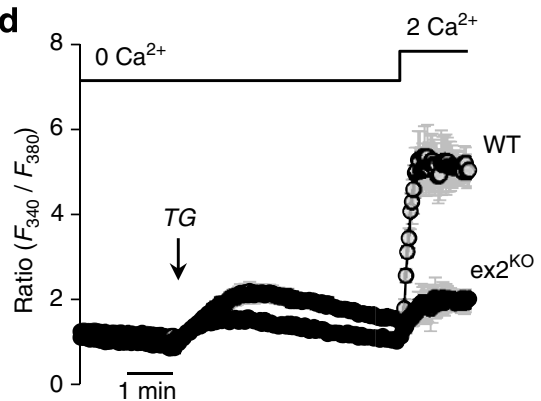

e

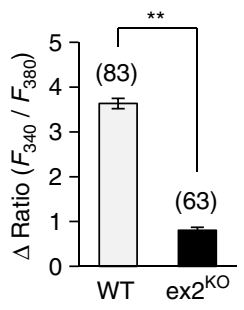

$\mathbf{f}$

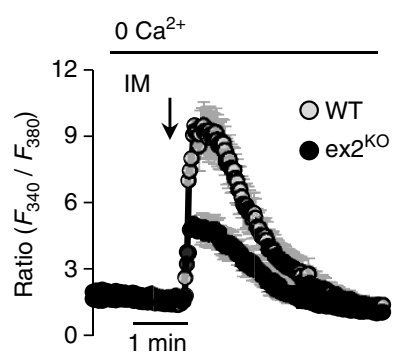

(Fig. 2f,g). The PLA2g6 ex $2^{\mathrm{KO}}$ mouse model offers a unique tool to determine if and how such cellular deficiencies could lead to PD pathology.

iPSC-derived DA neurons from PLA2g6 ex2 $2^{\mathrm{KO}}$ and WT mice. The role of SOCE in live DA neurons is obscure, so the next two questions were (i) whether SOCE is present in DA neurons and (ii) if DA neurons from PLA2g6 ex $2^{\mathrm{KO}}$ mice had similar defects in SOCE and $\mathrm{ER} \mathrm{Ca}^{2+}$, as we found in fibroblasts. To assess the role of PLA2g6 and SOCE in live DA neurons, an induced pluripotent stem cell (iPSC) approach was used to create iPSC-derived A9 midbrain DA neurons ${ }^{42}$ from WT and PLA2g6 ex2 ${ }^{\mathrm{KO}}$ MEFs (see Methods and Supplementary Fig. 12).

Live-cell $\mathrm{Ca}^{2}+$ imaging (Fig. 3a,b) revealed that iPSC-derived tyrosine hydroxylase-positive $(\mathrm{TH}+) \mathrm{DA}$ neurons indeed have classical TG-induced $\mathrm{Ca}^{2+}$ responses (Fig. 3c), including passive release of $\mathrm{Ca}^{2+}$ from the stores and SOCE that could be detected on $\mathrm{Ca}^{2}+$ re-addition. These data present first evidence for SOCE in iPSC-derived DA neurons. Notably, the amplitude of SOCE in DA neurons appeared to be very small compared with SOCE in MEFs under the same experimental conditions: on $\mathrm{Ca}^{2+}$ addition to TG-treated cells $\Delta$ Ratio was $0.31 \pm 0.18$ in DA neurons $(n=12)$ versus $2.39 \pm 0.16(n=31)$ in MEFs. Similarly, ionomycin-induced $\mathrm{Ca}^{2+}$ release in DA neurons (Fig. 3e,f) also appeared to be significantly smaller than in MEFs: $\Delta$ Ratio $=0.87 \pm 0.03(n=11)$ in DA neurons versus $2.66 \pm 0.21$ $(n=26)$ in MEFs. Small $\mathrm{Ca}^{2+}$ release was consistent with previously acknowledged low $\mathrm{Ca}^{2+}$ buffering capacity of intracellular $\mathrm{Ca}^{2+}$ stores in DA neurons of substantia nigra pars compacta $(\mathrm{SNc})^{43}$.

Further, we found that SOCE and ionomycin-induced $\mathrm{Ca}^{2+}$ store release are significantly impaired in DA neurons from PLA2g6 ex $2^{\mathrm{KO}}$ mice (Fig. 3c-f), emulating the results obtained in MEF cells. Thus, impairment of PLA2g6 translates into significant loss of apparently limited store-operated $\mathrm{Ca}^{2}+$ signalling in DA neurons, which could make these cells particularly vulnerable to PLA2g6 and SOCE dysfunction.

\section{Figure 2 | New PARK14 (PLA2g6) ex2 ${ }^{\mathrm{KO}}$ mouse model mimics} idPD-associated deficiency in store-operated PLA2g6-dependent $\mathrm{Ca}^{2+}$ signalling. (a) Schematic illustration of Pla2g6 (PARK14) gene with exons and ATG sites; below, corresponding full-length ( $L$ ) variant of WT PLA2g6 protein and $\mathrm{N}$-terminal truncated protein in ex $2^{\mathrm{KO}}$ mouse in which exon 2 (in red) was genetically deleted (Supplementary Figs 5-9). (b) Catalytic activity of PLA2g6 in homogenates of MEFs from WT and ex $2^{\mathrm{KO}}$ mice: summary results show average activity ( \pm s.e.) from three repetitions under basal conditions, after activation in the presence of $10 \mathrm{mM}$ EGTA and after inhibition with $25 \mu \mathrm{M}$ (S)-BEL. (c) Activation of PLA2g6 by TG-induced depletion of $\mathrm{Ca}^{2+}$ stores in intact MEFs from WT and ex2 ${ }^{\mathrm{KO}}$ mice: summary results show average activity ( \pm s.e. from three repetitions) under basal conditions, after activation by TG ( $5 \mu \mathrm{M}$ for $10 \mathrm{~min})$ and after inhibition with (S)-BEL. (d) Impairment of TG-induced SOCE in the ex $2^{\mathrm{KO}}$ MEFs: representative traces show $\mathrm{Ca}^{2}+$ response to TG $(5 \mu \mathrm{M})$ application in the absence of extracellular $\mathrm{Ca}^{2+}$, followed by SOCE on $\mathrm{Ca}^{2+}$ addition in WT and ex $2^{\mathrm{KO}}$ cells. Each trace shows $\mathrm{Ca}^{2}+$ responses (average \pm s.d.) in a group of 10-20 individual cells measured simultaneously. (e) Summary data show the differences in the peak SOCE in the WT and ex $2^{\mathrm{KO}}$ cells: average \pm s.e. from 3-6 independent experiments per each condition. (f) Representative traces (average \pm s.d.) show ionomycin (IM, $1 \mu \mathrm{M}$ )induced $\mathrm{Ca}^{2}+$ release from intracellular stores (in the presence of extracellular EGTA) in WT and ex $2^{\mathrm{KO}}$ MEFs. (g) Summary data from experiments like in $\mathbf{f}$ show peak $\mathrm{Ca}^{2}+$ release (average \pm s.e.) from 3-6 independent experiments. The numbers of cells summarized for each condition is specified above the bars; ${ }^{\star \star} P<0.01$. 
a
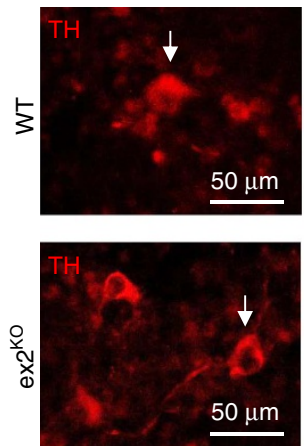

C

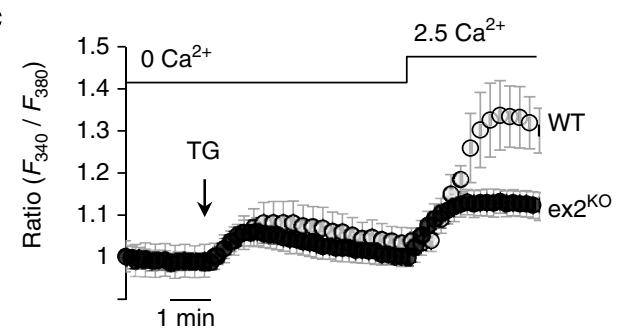

e

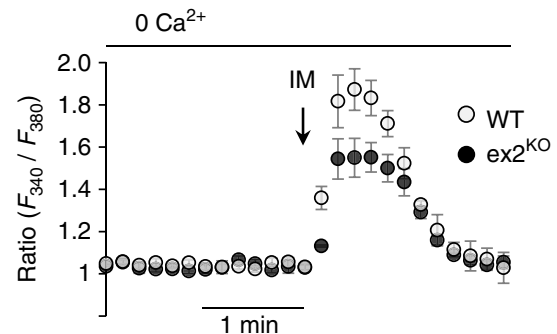

b Fura-2 ratio
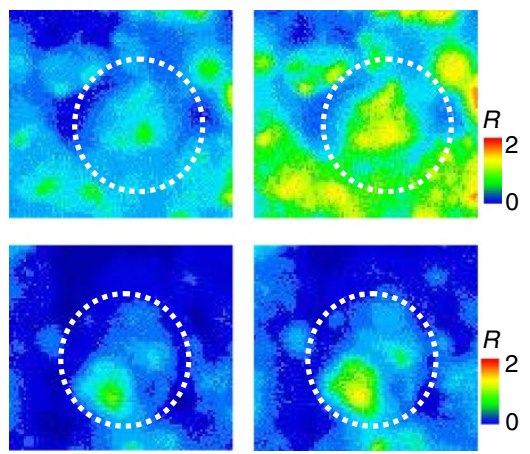

d

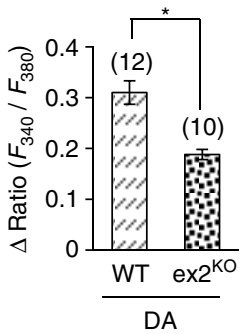

$\mathbf{f}$

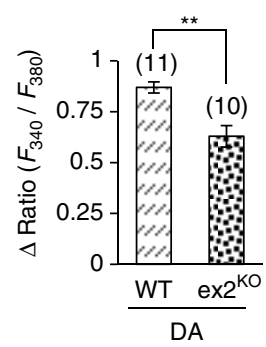

Figure 3 | Store-operated $\mathbf{C a}^{\mathbf{2}}+$ signalling in iPSC-derived DA neurons from WT and ex2 $\mathbf{2}^{\mathbf{K O}}$ mice. (a) Representative images of iPSC-derived A9 midbrain DA neurons positive for TH $\left(\mathrm{TH}+\right.$ ) from WT and PLA2g6 ex $2^{\mathrm{KO}}$ mice (see Supplementary Fig. 12 for more details); (b) images demonstrate $\mathrm{Ca}^{2}+$ changes due to SOCE in specific DA neurons outlined by dotted circles and shown by an arrow in a: images show Fura-2 Ratio $\left(F_{340} / F_{380}\right)$ in individual TH + neuron before (left) and after (right) $\mathrm{Ca}^{2+}$ addition to TG-pretreated cells, as shown in c. (c) TG-induced SOCE (average \pm s.d.) in individual iPSC-derived DA $(\mathrm{TH}+)$ neurons from WT and ex $2^{\mathrm{KO}}$ mice: traces show $\mathrm{Ca}^{2}+$ changes in response to TG $(5 \mu \mathrm{M})$ application in the absence of extracellular $\mathrm{Ca}^{2}+$, followed by SOCE on $\mathrm{Ca}^{2+}$ addition. (d) Summary data comparing the peak SOCE (average \pm s.e.) in DA (TH + ) neurons from WT and ex2 $2^{\mathrm{KO}}$ mice. (e) lonomycin ( $\mathrm{IM} ; 100 \mathrm{nM}$ )-induced $\mathrm{Ca}^{2}+$ release (average \pm s.d.) from intracellular stores in DA $\left(\mathrm{TH}+\right.$ ) neurons from $\mathrm{WT}$ and ex2 ${ }^{\mathrm{KO}}$ mice. (f) Summary data from experiments like in $\mathbf{f}$ show peak $\mathrm{Ca}^{2}+$ release (average \pm s.e.). The data represent the results from three independent experiments. The numbers of cells analysed for each condition is specified above the bars; ${ }^{\star} P<0.05$, ${ }^{\star \star} P<0.01$.

PLA2g6 ex2 ${ }^{\mathrm{KO}}$ mice develop age-dependent PD-like phenotype. Ageing is a primary risk factor for idPD ${ }^{44}$, and strikingly, all homozygous PLA2g6 ex $2^{\mathrm{KO}}$ mice (males and females) developed progressive age-dependent motor dysfunction (Fig. 4a) at an age range that aligns with that typical of idPD in humans. WT and heterozygous littermates remained normal throughout the same observation period, consistent with autosomal recessive inheritance of PARK14 (PLA2g6)-associated fPD in humans. Analysis of the SNc area of the brain from aged animals revealed a significant increase in the number of degenerative periodic acid-Schiff (PAS)-positive neurons (Fig. 4b) in the $\mathrm{ex}^{\mathrm{KO}}$ animals, and Nissl staining suggested potential loss of DA neurons (Supplementary Fig. 13). The results of the blinded stereological analysis of $\mathrm{SNc}$ confirmed progressive agedependent loss of $\mathrm{TH}+\mathrm{DA}$ neurons (Fig. 4c,d and Supplementary Figs 13 and 14): while the number of $\mathrm{TH}+$ neurons was the same in 8 -month-old ex $2^{\mathrm{KO}}$ and WT animals (consistent with no motor dysfunction in this preclinical stage), over $30 \%$ of DA neurons in SNc of ex 2 KO mice was lost by 16 months (early clinical) and over 50\% was lost by 24 months of age (late clinical stage). Notably, analysis of the hippocampus and
M1/M2 areas of the temporal cortices revealed no signs of neurodegeneration in ex $2^{\mathrm{KO}}$ animals (Fig. 4b), demonstrating that motor dysfunction and DA neuronal loss in SNc was not the result of a widespread nonspecific neurodegeneration.

Progressive loss of DA neurons in $\mathrm{SNc}$ is known to be a major factor in motor dysfunction in human $\mathrm{PD}^{45}$. Similarly, ex $2^{\mathrm{KO}}$ animals developed a strong age-dependent PD-like motor dysfunction, which was validated using an array of the standard behavioural tests (Fig. 4e-i). The balance beam test (Fig. 4e) showed impairment of motor coordination and progressive agedependent increase in the number of missteps made by ex $2^{\mathrm{KO}}$ mice, with no change in performance of ageing WT animals. Importantly, the DA nature of motor dysfunction was confirmed by the L-DOPA test (Fig. 4f): administration of L-DOPA markedly improved motor coordination of ex $2^{\mathrm{KO}}$ animals in age- and dose-dependent manner, which was similar to L-DOPA effects in humans ${ }^{46}$. Figure $4 \mathrm{f}$ shows that while the lowest dose of L-DOPA ( $5 \mathrm{mg}$ per $\mathrm{kg}$ body weight) produced a marked improvement in the balance beam performance $(60 \%$ reduction in the number of missteps) of the 12 -month-old ex $2^{\mathrm{KO}}$ animals (early clinical stage), significantly higher doses were required to produce similar 
a

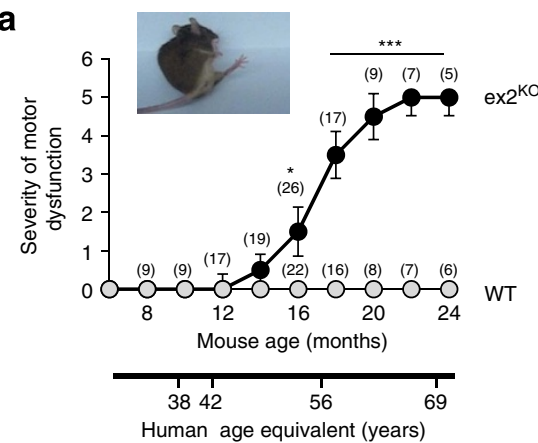

C

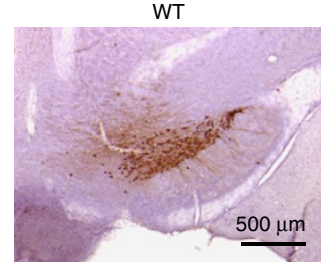

WT

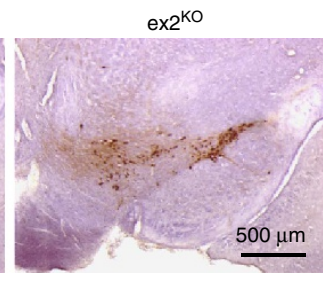

b

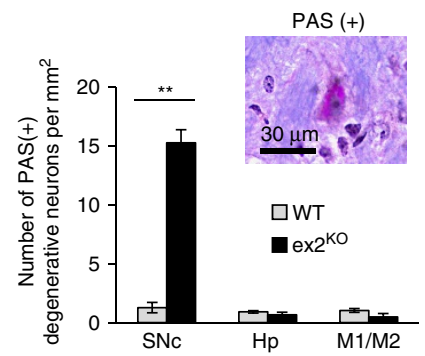

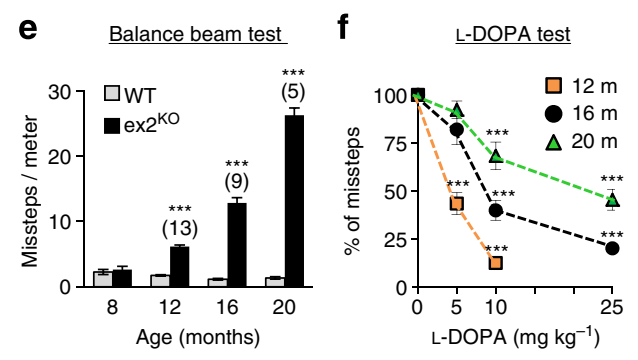
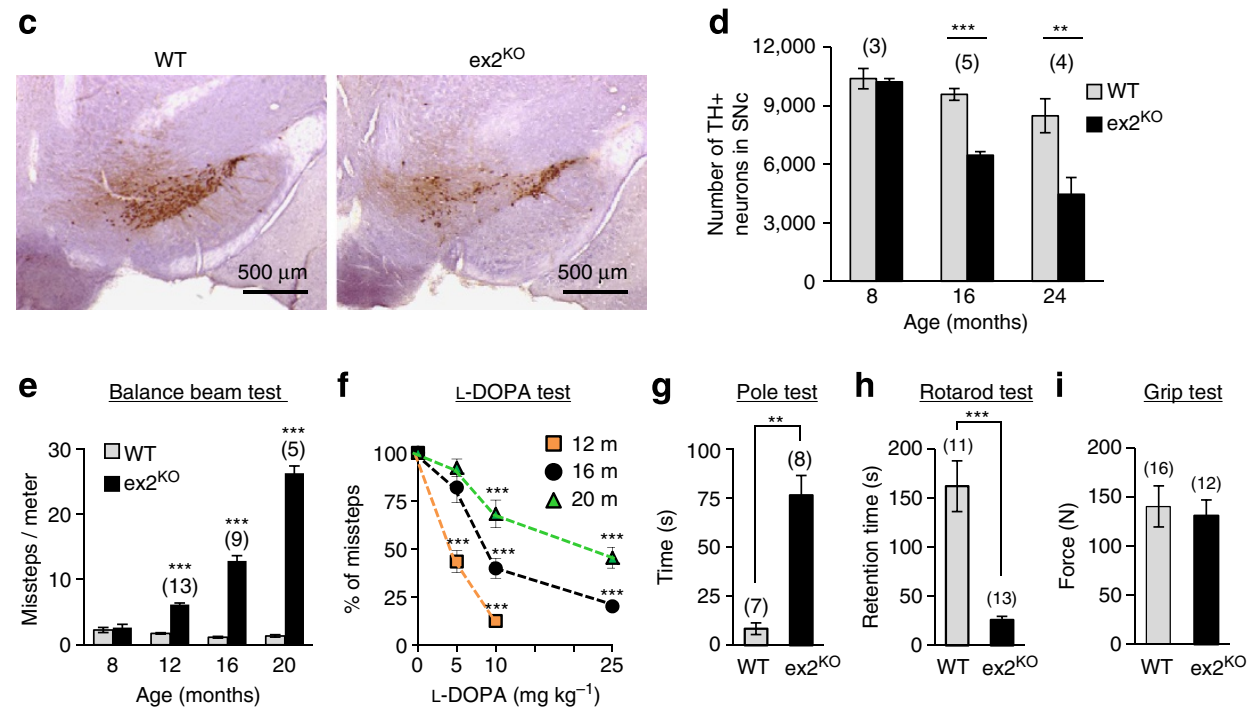

Figure 4 | Progressive loss of DA neurons and age-dependent PD-like motor dysfunction in ex $\mathbf{2}^{\mathbf{K O}}$ mice. (a) progressive motor dysfunction in ageing ex $2^{\mathrm{KO}}$, but not in WT mice (see Methods for details); human age equivalent is shown below; inset illustrates unstable gait of representative 18-month-old ex $2^{\mathrm{KO}}$ animal; numbers above each point represent the number of animals per each age group. (b) Analysis of PAS staining shows significant increase in the number of PAS $(+)$ degenerative neurons in $\mathrm{SNc}$, but not in hippocampus $(\mathrm{Hp})$ or in $\mathrm{M} 1 / \mathrm{M} 2$ motor cortex of the $16-\mathrm{month}-\mathrm{old}$ ex $2^{\mathrm{KO}}$ mice (average \pm s.e. from three pairs of age-matched animals). (c) Example of immunostaining of $\mathrm{TH}+$ (brown) neurons in $\mathrm{SNc}$ in the brain from $\mathrm{WT}$ and ex $2^{\mathrm{KO}}$ littermates (16 months old); scale bar, $500 \mu \mathrm{m}$. (d) Summary data (average \pm s.e.) show progressive age-dependent reduction in the number of TH + neurons in ex $2^{\mathrm{KO}}$ mice; the results of the blinded stereological analysis of $\mathrm{SN}$ area of the brain in the groups of age-matched WT and ex $2^{\mathrm{KO}}$ animals (for more details see Supplementary Fig. 13). (e-i) Behavioural studies of the age-matched groups of the WT and ex2 ${ }^{\mathrm{KO}}$ mice show: (e) progressive agedependent increase in the number of missteps in the balance beam test; (f) age- and dose-dependent improvement of motor performance by L-DOPA in ex $2^{\mathrm{KO}}$ mice; relative change in the number of missteps in balance beam test made $1 \mathrm{~h}$ after L-DOPA (5, 10 or $\left.25 \mu \mathrm{M}\right)$ administration versus control in the 12 $(n=12)$-, $16(n=8)$ - and $20(n=8)$-month-old animals; ( $(\mathbf{s})$ significant increase in time required for 16 -month-old ex $2^{\mathrm{KO}}$ mice to reorient on the top of the pole; (h) significantly reduced rotarod performance of the 16- to 18-month-old ex $2^{\mathrm{KO}}$ mice; (i) no difference in the grip strength of the 16- to 18-month-old $\mathrm{WT}$ and ex $2^{\mathrm{KO}}$ mice. All data are mean \pm s.e.; numbers above each bar represent the number of animals tested per each group; ${ }^{\star \star} P<0.01$, ${ }^{\star \star \star} P<0.001$.

effects at more advanced clinical stages in 16- and 20-month-old animals. The pole (Fig. 4g) and rotarod tests (Fig. 4h) further confirmed significant PD-like motor dysfunction in ex $2^{\mathrm{KO}}$ animals. Grip test (Fig. 4i) showed no difference between WT and $\mathrm{ex}_{2} \mathrm{KO}$ animals, thus demonstrating that motor dysfunction in ex $2^{\mathrm{KO}}$ animals is not caused by the loss of the strength in their limbs.

Thus, PLA2g6 ex2 $2^{\mathrm{KO}}$ mouse model presents progressive loss of DA neurons in SNc and age-dependent L-DOPA-sensitive PD-like motor dysfunction, which mimics idPD in ageing humans.

Autophagic dysfunction in DA neurons of PLA2g6 ex $2^{\mathrm{KO}}$ mice. The question arises, how does impairment of store-operated $\mathrm{Ca}^{2+}$ signalling lead to demise of DA neurons in SNc? More specifically, could disruption in store-operated $\mathrm{Ca}^{2+}$ signalling trigger some cellular pathology that is already established as a hallmark of human PD? Analysis of the SNc area of the brain in ex $2^{\mathrm{KO}}$ mice revealed that PLA2g6(L) protein is highly expressed in specific $\mathrm{TH}+\mathrm{DA}$ neurons (Fig. 5a and Supplementary Fig. 15), suggesting that $\mathrm{DA}$ neurons in $\mathrm{SNc}$ may have a particularly high demand for PLA2g6(L), and impairment of the PLA2g6-dependent SOCE function (Fig. 3) may be particularly stressful for these neurons. Closer analysis of $\mathrm{TH}+$ neurons in SNc of PLA2g6 ex $2^{\mathrm{KO}}$ mice revealed that DA neurons experience significant autophagic dysfunction. Figure $5 \mathrm{~b}$ and Supplementary Fig. 16 show that TH + neurons in SNc in ex $2^{\mathrm{KO}}$ have significant accumulation of LC3 (microtubuleassociated protein $1 \mathrm{~A} / 1 \mathrm{~B}$-light chain 3 , established marker of autophagic flux), which is not found in WT animals. The increased autophagosome numbers in $\mathrm{ex}^{\mathrm{KO}}$ mice, which can result from impaired autophagic flux, was also manifested by a significant increase in the ratio of LC3-II/actin (Fig. 5c). Thus, in vivo $\mathrm{DA}$ neurons in the $\mathrm{SNc}$ of PLA2g6-deficient $\mathrm{ex} 2{ }^{\mathrm{KO}}$ mice 

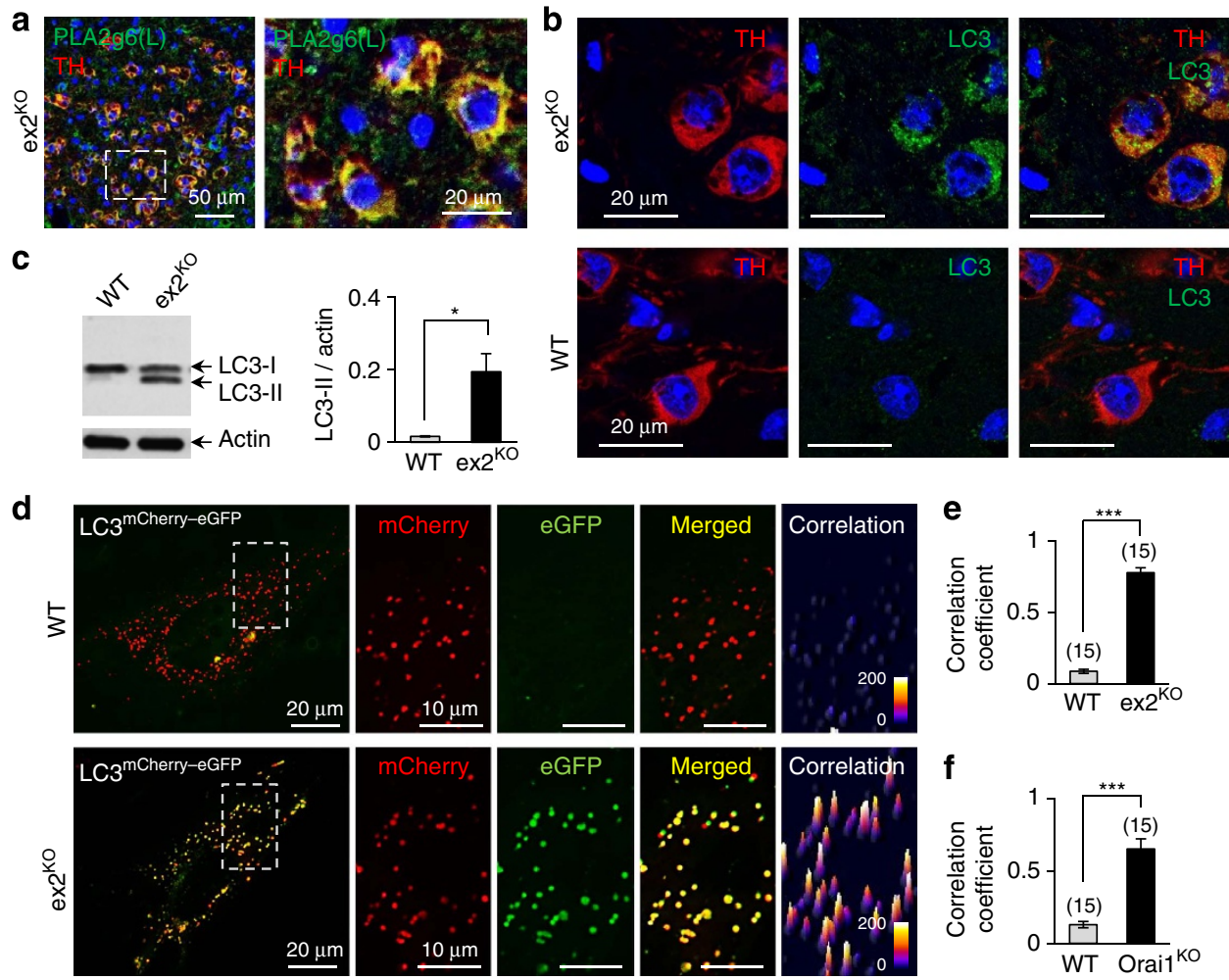

Figure 5 | Autophagic dysfunction in ex $2^{\mathrm{KO}}$ mice. (a) Co-localization of TH (red) and PLA2g6(L) (green) in DA neurons in SNc of ex $2^{\mathrm{KO}}$ mice (see Supplementary Fig. 14 for details). Nuclei are stained with DAPI (blue). Image on the right shows magnified part of the image on the left (identified by dotted rectangle). (b) Representative images show LC3 aggregation in $\mathrm{TH}+$ neurons of the ex $2^{\mathrm{KO}}$, but not WT brain: results of co-immunostaining for TH (red), LC3 (green) and DAPI (blue) in SNc area of the brain from 16-month-old ex $2^{\mathrm{KO}}$ and WT littermates (see Supplementary Fig. 15 for details). (c) Representative western blot (WB) and summary data (average \pm s.e.) show significant increase in LC3-II/actin ratio in tissue samples from the ex $2^{\mathrm{KO}}$ mice $(n=3)$. Images have been cropped for presentation. Full-size images are shown in Supplementary Fig. 22. (d) Representative images of LC $3^{\text {mCherry-eGFP }}$ (tandem mCherry (red)-eGFP (green)-tagged LC3) in live MEF cells from the WT and ex $2^{\mathrm{KO}}$ mice. Composite image of the whole cell is shown on the left, and magnified mCherry, eGFP and merged images on the right show the part of the cell identified by dotted rectangle. Far right image shows correlation map for red and green signals (see Supplementary Fig. 17 and Methods for details). (e) Summary data show increase in the correlation coefficient (in experiments like in $\mathbf{d}$ ) in the cells from ex $2^{\mathrm{KO}}$ mice, compared with WT cells; bars show average ( \pm s.e.) from three independent experiments. (f) Summary data like in e show significant increase in the correlation coefficient in the cells from Orai1 ${ }^{\mathrm{KO}}$ mice (for more details see Supplementary Fig. 18). ${ }^{\star} P<0.05,{ }^{\star \star \star} P<0.001$.

may experience marked autophagic dysfunction, which is one of the major hallmarks of human $\mathrm{PD}^{47-51}$.

Causal link between PLA2g6, SOCE, ER $\mathrm{Ca}^{2+}$ and autophagy. To better understand a previously unknown association of PLA2g6-dependent $\mathrm{Ca}^{2+}$ signalling with autophagy, primary MEFs from WT and ex $2^{\mathrm{KO}}$ mice were used as a model for livecell imaging and molecular rescue studies. Using a tandem tagged LC3 $3^{\text {mCherry-eGFP }}$ as a marker of autophagic flow ${ }^{52}$ we confirmed significant autophagic dysfunction in the cells from ex $2^{\mathrm{KO}}$ animals. Image analysis of MEFs expressing LC3 ${ }^{\text {mCherry-eGFP }}$ (Fig. 5d) revealed that in WT cells this marker successfully reaches lysosomes, where the eGFP (but not mCherry) signal is quenched by the high acidic environment, resulting in a loss of green fluorescent protein (GFP) fluorescence. In contrast, the enhanced GFP (eGFP) signal remained very prominent and spatially co-localized with mCherry in autophagosomes of ex $2^{\mathrm{KO}}$ cells. Correlation analysis of mCherry and eGFP fluorescence confirmed very significant differences between ex $2^{\mathrm{KO}}$ and WT cells: in ex $2^{\mathrm{KO}}$ cells, the fluorescent signals were strongly correlated (Fig. 5e) and the size of LC3-containing particles was larger (Supplementary Fig. 17) than in WT cells. Autophagic arrest in ex $2^{\mathrm{KO}}$ cells resembled the effects of prolonged TG treatment in WT MEFs (Supplementary Fig. 17), which was consistent with recent report ${ }^{53}$ of TG-induced autophagic arrest due to impairment of autophagosome fusion with lysosomes. There is also a possibility that fusion may occur, but lysosomal acidification may be defective.

Interestingly, a similar autophagic dysfunction was also produced by the targeted deletion of the Orail channel (Fig. $5 f$ ), which is a critical component of SOCE machinery located downstream from PLA2g6 (refs 25,26). Supplementary Fig. 18 demonstrate that MEF cells from Orai1 ${ }^{\mathrm{KO}}$ mice ${ }^{32,54}$ have significant impairment of SOCE, depletion of ER $\mathrm{Ca}^{2+}$ stores and autophagic dysfunction, which closely mimic deficiencies in PLA2g6 ex2 $2^{\mathrm{KO}}$ cells. Thus, similar autophagic arrest can be produced by genetic deletion of Orail, or impairing PLA2g6 activation, or inhibiting SERCA (Sarco/ endoplasmic rReticulum $\mathrm{Ca}^{2}+$ ATPase)-dependent refilling of the stores with TG. Remarkably, while all these interventions cause depletion of $\mathrm{ER} \mathrm{Ca}^{2+}$ stores, only Orai1 ${ }^{\mathrm{KO}}$ and $\mathrm{ex} 2^{\mathrm{KO}}$ inhibit SOCE, while TG activates it. Thus, depletion of $\mathrm{Ca}^{2}+$ stores (rather than simple loss of SOCE) seems to be a most likely trigger for autophagic dysfunction.

To verify a causative role of PLA2g6 in impairment of the store-operated $\mathrm{Ca}^{2+}$ signalling and autophagic dysfunction, and to further link it to human $\mathrm{PD}$, two molecular approaches were 

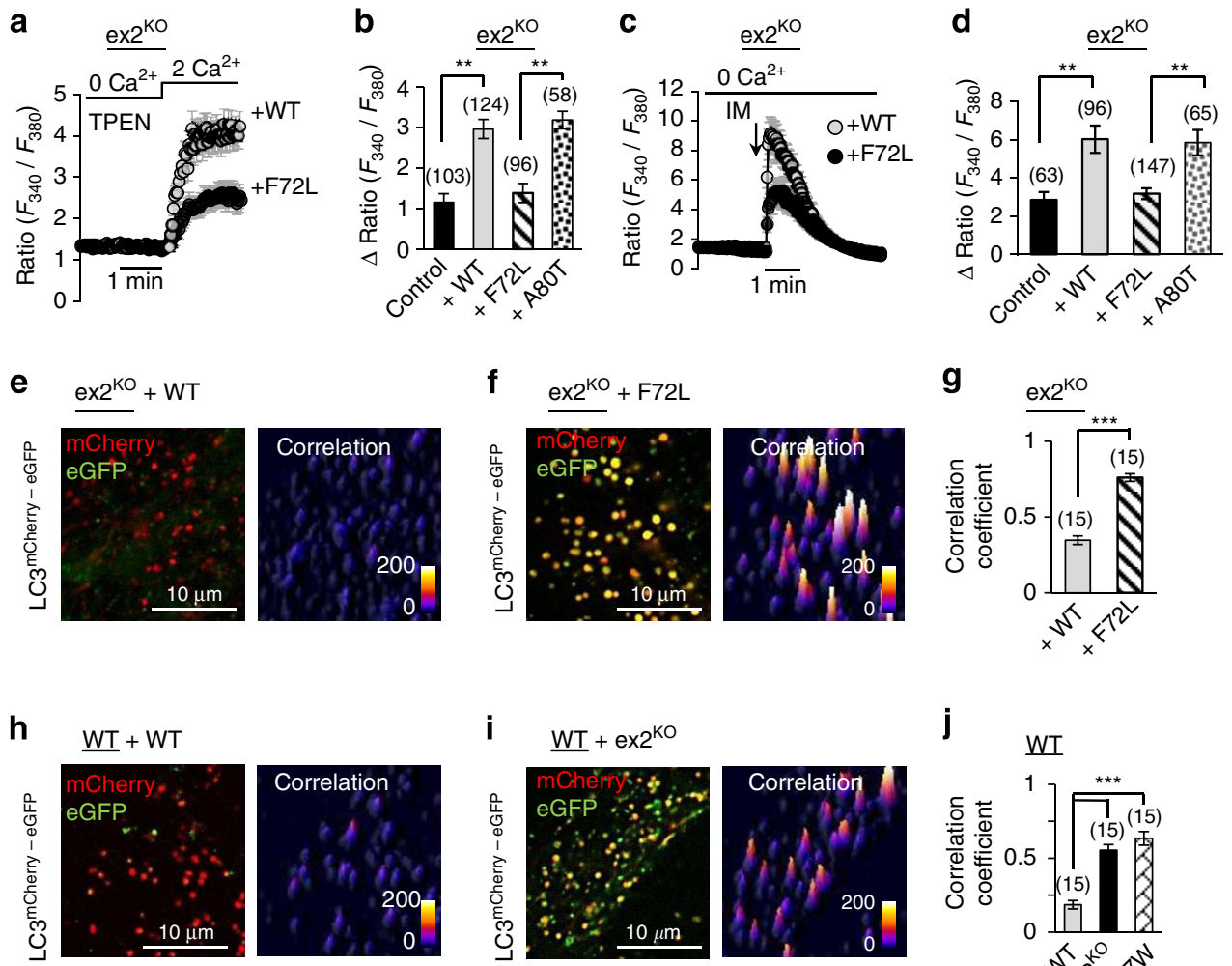

\section{j}
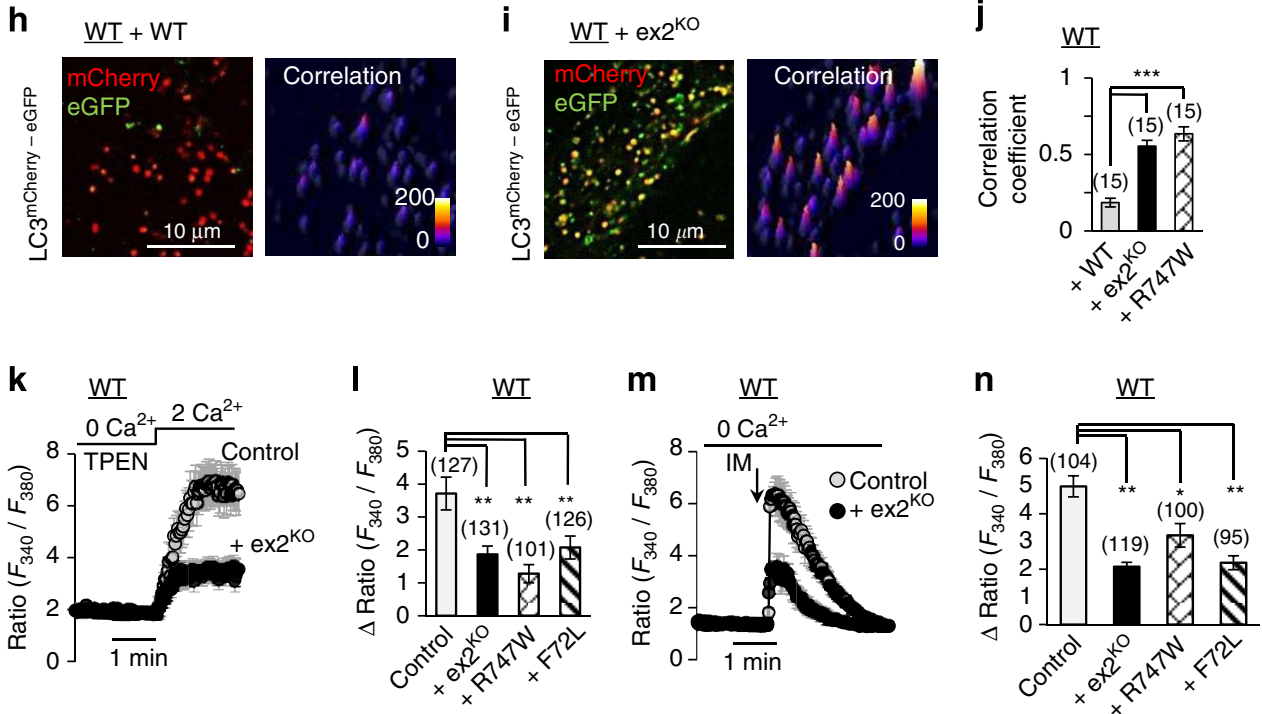

Figure 6 | Causal relationship between PLA2g6-dependent $\mathrm{Ca}^{2}+$ signalling and autophagic dysfunction, and its relevance to human PD. (a-g) Rescue experiments in live ex2 $2^{\mathrm{KO}} \mathrm{MEF}$ cells transfected with WT PLA2g6(L) or its PD-associated F72L mutant, or A80T mutant that does not have association with human PD. (h-n) Dominant-negative effects of PLA2g6(L) deficiency in live WT MEF cells transfected with empty vector (control) or PLA2g6(L) ex $2^{\mathrm{KO}}$ or one of two human PD-associated PLA2g6(L) mutants (F72L or R747W). (a,b,k,l), SOCE on Ca ${ }^{2}+$ addition to TPEN-pretreated cells (400 $\mu M$ for

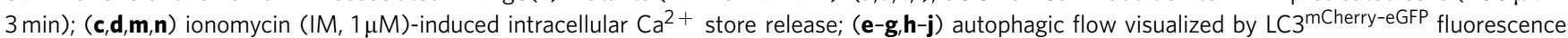
(as in Fig. $4 d-e$ ) in live MEF cells. Representative $\mathrm{Ca}^{2+}$ traces show average \pm s.d. from 10-20 cells recorded simultaneously. All summary data (bars) show average \pm s.e. from 3-6 independent experiments per each condition; the number of cells for each condition is specified above the bars; ${ }^{\star} P<0.05$, ${ }^{\star \star} P<0.01,{ }^{\star \star \star} P<0.001$. For more details see Supplementary Figs 19 and 20.

used. First, molecular rescue experiments were performed in ex2 ${ }^{\mathrm{KO}}$ MEFs (Fig. 6a-g) to determine if expression of WT PLA2g6 could recover normal store-operated $\mathrm{Ca}^{2+}$ signalling and autophagic function. Second, we tested if expression of a PLA2g6 mutant that is associated with human fPD could impair normal function of WT cells (Fig. 6h-n).

We found that deficient SOCE and depleted $\mathrm{Ca}^{2+}$ stores in ex $2^{\mathrm{KO}}$ cells can be effectively rescued by simple expression of the functional PLA2g6(L) (Fig. 6a,d). Restoration of SOCE and $\mathrm{Ca}^{2+}$ stores by WT PLA2g(L) was also sufficient to rescue autophagic flow and restore processing of LC3 ${ }^{\text {mCherry-eGFP }}$ (Fig. 6e,g and Supplementary Fig. 19). Remarkably, expression of PLA2g6(L) that carries human fPD mutation ${ }^{6}$ (F72L, located in the $\mathrm{N}$ terminus of PLA2g6) failed to recover SOCE, or refill $\mathrm{Ca}^{2+}$ in the stores, and did not restore autophagic function (Fig. 6a-g and Supplementary Fig. 19). In contrast, the A80T mutant, which is not associated with human PD was able to fully restore normal $\mathrm{Ca}^{2+}$ signalling function (Fig. 6b,d).

To test if human PD-associated mutations in PLA2g6/PARK14 indeed may cause targeted impairment of the store-operated $\mathrm{Ca}^{2+}$ signalling leading to autophagic dysfunction, the effects of acute expression of F72L and R747W mutants of PLA2g6(L) were tested in WT MEFs. Figure 6k-n demonstrate that these PD-associated mutants can indeed cause impairment of SOCE, depletion of the stores and impairment of autophagic flux. Importantly, the pathological effects of human PD-associated mutants were identical to those produced by expression of the $\mathrm{N}$-terminally truncated PLA2g6(L) that mimics ex $2^{\mathrm{KO}}$ deficiency in our mouse model: expression of $\mathrm{ex}^{\mathrm{KO}}$ protein effectively impaired SOCE (Fig. 6k,l), depleted $\mathrm{Ca}^{2+}$ stores (Fig. 6m,n) and lead to significant autophagic dysfunction (Fig. $6 \mathrm{~h}-\mathrm{j}$ and Supplementary Fig. 20). The effects caused by the overexpression 
of these mutants were similar to what we found in MEFs from ex $2^{\mathrm{KO}}$ mice (Figs $2 \mathrm{~d}-\mathrm{g}$ and $4 \mathrm{~d}, \mathrm{e}$ ), and in fibroblasts from the $\mathrm{fPD}^{\mathrm{R} 747 \mathrm{~W}}$ patient. Thus, targeted impairment or restoration of PLA2g6 protein indeed can, respectively, impair or rescue store-operated $\mathrm{Ca}^{2}+$ signalling and autophagic function, further demonstrating direct association and leading role of PLA2g6 in these cellular events.

PLA2g6(L) can rescue SOCE and autophagy in idPD cells. The results of the genetic and molecular manipulations with PLA2g6 validated a causal link between PLA2g6 deficiency, store-operated $\mathrm{Ca}^{2+}$ signalling and autophagy, and demonstrated that two PLA2g6(L) mutations associated with human fPD can cause impairment in SOCE, depletion of the stores and autophagic dysfunction. To determine if a similar sequence of pathological events could be associated with idPD, live-cell imaging and molecular rescue experiments were performed in primary human skin fibroblasts from idPD and control patients.

Analysis of primary skin fibroblasts from idPD and $\mathrm{fPD}^{\mathrm{R} 747 \mathrm{~W}}$ patients revealed that in addition to reduced SOCE and depleted stores (Fig. 1), they all have significant autophagic dysfunction, as demonstrated by significant impairment of LC $3^{\text {mCherry-eGFP flow }}$ (Fig. 7a,b and Supplementary Fig. 21). Thus, impairment of SOCE, depletion of ER $\mathrm{Ca}^{2+}$ stores and autophagic dysfunction (Fig. 7a,b) represent a new distinct sequence of pathological cellular events that could be found not only in DA neurons and MEFs from PLA2g6 ex $2^{\mathrm{KO}}$ mice but also in primary skin fibroblasts from idPD and $\mathrm{PPD}(P A R K 14)$ patients.

If specific defects in PLA2g6(L) expression (Fig. 1f) and/or store-dependent activation (Fig. 1e) could indeed be a major cause of SOCE deficiency and autophagic dysfunction in idPD cells, one would expect that overexpression of functional WT PLA2g6(L) should rescue SOCE and improve autophagic flux in idPD cells. Remarkably, the results of rescue experiments presented in Fig. $7 \mathrm{c}, \mathrm{d}$ demonstrate that this prediction is correct, and PLA2g6(L) expression can indeed significantly improve SOCE and autophagic flux in idPD cells.

Altogether, the results in primary cells from idPD and $\mathrm{fPD}^{\mathrm{R} 747 \mathrm{~W}}$ patients confirmed the critical role of PLA2g6(L) in impaired SOCE and autophagic dysfunction, and validated previously unknown association of these events with human idPD and PARK14 fPD.
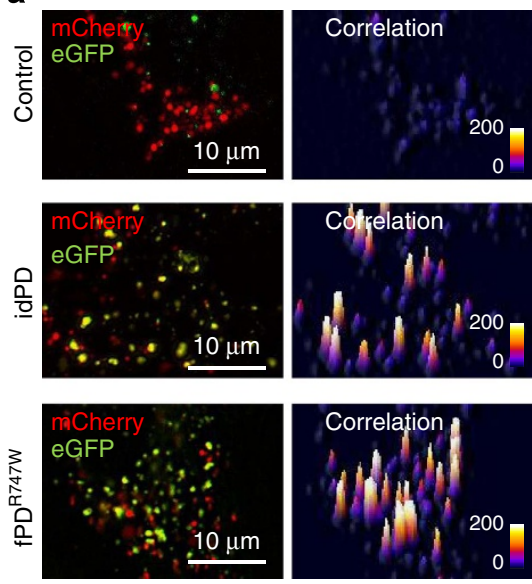

b

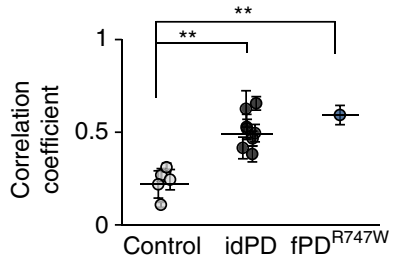

C

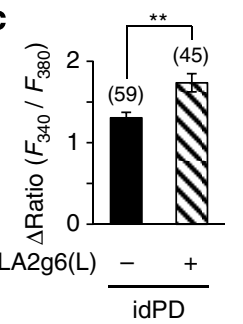

d

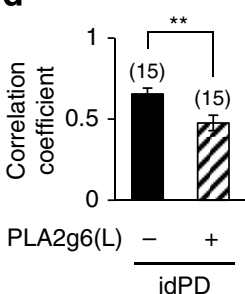

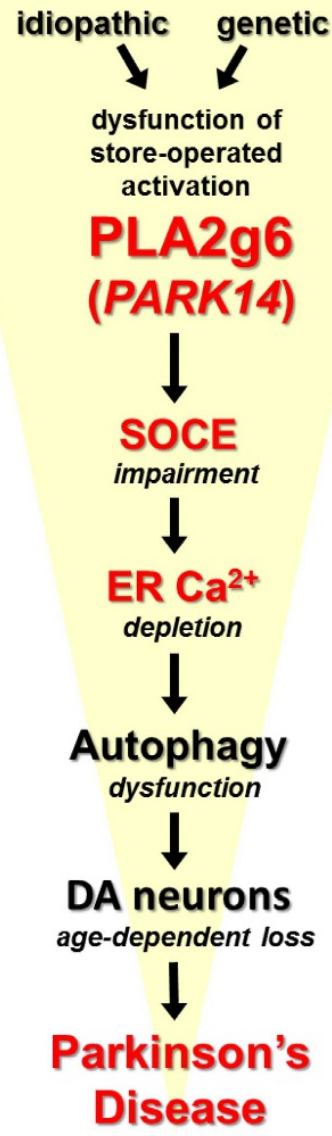

Figure 7 | PLA2g6 (PARK14)-dependent $\mathbf{C a}^{2}+$ signalling as a novel determinant of PD. (a) Representative images and correlation maps of LC ${ }^{\mathrm{mCherry}-\mathrm{eGFP}}$ in live primary human skin fibroblasts from control, idPD and fPD PLA2g6 R747W mutant patients. (b) Summary data (average \pm s.e.) from experiments like in a show significant impairment of autophagy in idPD $(n=10)$ and fPD ${ }^{R 747 W}(n=1)$ versus control $(n=5)$ patients, which is evident from the higher correlation coefficient for LC3 ${ }^{m C h e r r y-e G F P}$ (see Supplementary Fig. 21 for details); ${ }^{\star \star} P<0.01$. Results of the rescue experiments in live fibroblasts from idPD patient show (c) SOCE (like in experiments in Fig. 1a) and (d) correlation coefficient (like in experiments in Fig. 6a,b) $48 \mathrm{~h}$ after cells transfection with either PLA2g6(L) or empty vector as a control. Summary data show average \pm s.e. from the numbers of cells specified above the bars. ${ }^{\star \star} P<0.01$. (e) Schematic illustration of a previously unknown sequence of pathological events that can be initiated by idiopathic or genetic deficiency in store-operated activation of PLA2g6 (PARK14), which can lead to SOCE impairment, depletion of intracellular $\mathrm{Ca}^{2+}$ stores and autophagic dysfunction, which results in progressive loss of DA neurons in SNc and age-dependent PD. 


\section{Discussion}

On the basis of our findings, we propose that dysfunction of PLA2g6-dependent $\mathrm{Ca}^{2+}$ signalling could be a previously unknown mechanism contributing to the pathophysiology of human PD. Figure 7e illustrates a sequence of pathological events that could be initiated by dysfunction of the store-dependent activation of PLA2g6, which we found in idiopathic and genetically induced forms of PD. Defects in PLA2g6(L) activation by depleted stores can be due to PD-associated mutations (like in $\mathrm{fPD}^{\mathrm{R} 747 \mathrm{~W}}$ patient) or reduced expression (like in idPD patients) or cleavage of the $\mathrm{N}$ terminus (mimicked in ex $2^{\mathrm{KO}}$ mice), all of which were found to cause impairment of SOCE and depletion of ER $\mathrm{Ca}^{2}+$. It is tempting to speculate that the limited SOCE and low capacity of $\mathrm{Ca}^{2}+$ stores (Fig. 3) could make DA neurons particularly vulnerable to additional loss of SOCE found in idPD humans and PLA2g6 ex $2^{\mathrm{KO}}$ mice, thus setting the stage for their premature demise, which could be aggravated and accelerated by other factors that will be discussed below. Further, our studies present the unexpected discovery of a previously unknown ability of the defects in PLA2g6-dependent store-operated $\mathrm{Ca}^{2+}$ signalling to trigger autophagic dysfunction, premature loss of $\mathrm{DA}$ neurons in SNc and age-dependent Parkinsonism. This sequence of pathological events was validated in a novel PLA2g6 ex $2^{\mathrm{KO}}$ mouse model, which mimics deficient PLA2g6-dependent $\mathrm{Ca}^{2}+$ signalling and autophagic dysfunction found in idPD and $\mathrm{fPD}^{\mathrm{R} 747 \mathrm{~W}}$ patients. Importantly, such defects in ex $2^{\mathrm{KO}}$ mice were directly associated with a marked age-dependent phenotype, which exhibits selective loss of DA neurons in the SNc and L-DOPA-sensitive motor dysfunction resembling human PD. The anatomic selectivity of age-dependent neurodegeneration in this model appears to be the best yet observed for PD-associated genetic defects in murine models ${ }^{55,56}$.

Discovery of a novel causal relationship between impaired SOCE, depleted stores, autophagic dysfunction and PD-like pathology illuminates complex role of $\mathrm{Ca}^{2}+$ signalling in PD. It creates an interesting paradigm: insufficient $\mathrm{Ca}^{2+}$ entry through SOCE mechanism can be as detrimental to DA neurons as excessive $\mathrm{Ca}^{2+}$ entry through voltage-gated $\mathrm{Ca}_{\mathrm{V}} 1.3$ channels. This apparent duality has a simple explanation: distinct $\mathrm{Ca}^{2+}$ entry pathways regulate different cellular processes ${ }^{57}$, and apparently can be linked to different pathological hallmarks of PD. Pioneering studies from Surmeier's laboratory showed that excessive $\mathrm{Ca}^{2}+$ entry through $\mathrm{Ca}_{\mathrm{V}} 1.3$ channels is linked to mitochondrial oxidant stress ${ }^{58,59}$, so that the physiological pacemaker activity of this $\mathrm{Ca}^{2}+$ channel increases pathological vulnerability of DA neurons. In contrast, $\mathrm{Ca}^{2+}$ entry through PLA2g6-independent ${ }^{36}$ TRPC1 channels was recently shown ${ }^{60}$ to be protective in a MPP + (1-methyl-4-phenylpyridinium) model of DA neuronal cell death. Our current findings demonstrate that impairment of $\mathrm{Ca}^{2+}$ influx through the PLA2g6-dependent SOCE mechanism leads to depletion of $\mathrm{ER} \mathrm{Ca}^{2+}$ stores, autophagic dysfunction and premature death of DA neurons, suggesting that preservation of SOCE and refilling of ER $\mathrm{Ca}^{2+}$ stores is essential for DA neuronal health and survival.

Why deficiency in SOCE, depleted ER $\mathrm{Ca}^{2+}$ stores and autophagic dysfunction may be particularly detrimental to DA neurons? Several reasons can be of particular importance, starting with the new empirical data demonstrating rather limited SOCE and low capacity of ER Ca stores in live iPSC-derived A9 midbrain DA neurons. Poor store-dependent $\mathrm{Ca}^{2}+$ signalling can increase vulnerability of DA neurons to mitochondrial stress imposed by constant pacemaker activity of $\mathrm{Ca}_{\mathrm{V}} 1.3$ channels (highlighted by the work of Surmeier's group ${ }^{58,59}$ ). This stress can be further exacerbated by idiopathic or genetic defect in PLA2g6-dependent $\mathrm{Ca}^{2+}$ signalling and autophagic dysfunction (found in idPD and $\mathrm{fPD}^{\mathrm{R} 747 \mathrm{~W}}$ patients, and demonstrated in DA neurons from PLA2g6-deficient mice), which can push DA neurons over the threshold. Moreover, high demand for dopamine production makes DA neurons especially vulnerable to $\mathrm{ER} \mathrm{Ca}^{2+}$ store depletion and autophagic dysfunction, which altogether may explain premature age-dependent demise of the nigral DA neurons in idPD patients and PLA2g6 ex $2 \mathrm{KO}$ mice.

Interestingly, distinct defects in $\mathrm{Ca}^{2+}$ signalling were recently associated with Alzheimer's disease (AD) and Huntington's (HD) disease: in contrast to $\mathrm{PD}, \mathrm{ER} \mathrm{Ca}^{2}{ }^{+}$stores in $\mathrm{AD}$ and $\mathrm{HD}$ were found to be overfilled ${ }^{61,62}$. Moreover, Bezprozvany and colleagues reported that HD can be associated with TRPC1 overexpression and upregulation of TRPC1-dependent $\mathrm{Ca}^{2+}$ entry ${ }^{62}$. In contrast, $\mathrm{AD}$ can be associated with a significant reduction in synaptic expression of STIM2 leading to impairment of highly localized STIM2-dependent $\mathrm{Ca}^{2+}$ entry in mushroom spines and resulting in their $\operatorname{loss}^{61}$. Altogether, these findings suggest a rather high specificity of distinct $\mathrm{Ca}^{2}+$ signalling pathways for specific forms of neurodegeneration: pathological changes in expression or function of PLA2g6, Orai1, TRPC1, STIM1, STIM2 or other molecules can affect distinct mechanisms of $\mathrm{Ca}^{2}+$ entry and/or storage, and lead to pathological changes in neurons (or distinct neuronal structures) that are most vulnerable to each specific defect.

The results of genetic and molecular manipulations with distinct molecules involved in SOCE presented new clues for the causality of PLA2g6(L)-dependent $\mathrm{Ca}^{2}+$ signalling events leading to autophagic dysfunction. We found that genetic ablation of Orail (in MEFs from Orai1 ${ }^{\mathrm{KO}}$ mice) mimics impairment of SOCE, ER store depletion and autophagic dysfunction found in PLA2g6 ex $2^{\mathrm{KO}}$ MEFs. Similar autophagic dysfunction can be also produced by TG, which inhibits SERCA and depletes ER Ca ${ }^{2+}$ stores. However, in contrast to TG, which activates SOCE, there is a significant loss of SOCE in Orail ${ }^{\mathrm{KO}}$ and PLA2g6 ex $2{ }^{\mathrm{KO}}$ cells that have normal cytosolic $\mathrm{Ca}^{2+}$. Thus, PLA2g6-dependent depletion of $\mathrm{Ca}^{2+}$ stores (rather than actual reduction in SOCE) is most likely to be responsible for autophagic dysfunction, and loss of DA neurons in SNc leading to PD, as illustrated by the sequence of pathological processes proposed in Fig. 7e.

Impairment of the store-operated $\mathrm{Ca}^{2}+$ signalling might trigger or accelerate other pathological processes that are detrimental to DA neurons beyond the impairment of autophagy. For example, significant depletion of $\mathrm{ER} \mathrm{Ca}^{2}+$ stores can also cause ER stress ${ }^{63}$ and unfolded protein response that plays important role in neurodegeneration ${ }^{64-66}$. It is important to emphasize that since PLA2g6-dependent $\mathrm{Ca}^{2}+$ signalling appears to be upstream of both autophagy and the unfolded protein response, its deficiency in DA neurons can contribute to (or set the stage for) aggregation to human $\alpha$-synuclein and Lewy body formation, which is a diagnostic hallmark of $\mathrm{PD}^{47,50,51,64,67,68}$, and can be found in patients with familial PARK14 mutations ${ }^{7}$. The late life onset of idPD and the late onset of PD-like phenotype in PLA2g6 ex $2^{\mathrm{KO}}$ mouse model indicate that additional age-dependent processes participate in the final demise of DA neurons that are put at risk by insufficient store-operated $\mathrm{Ca}^{2+}$ signalling (Fig. 3). Oxidative stress, mitochondrial dysfunction and/or protein misfolding are the hallmarks of a normal ageing process ${ }^{44}$, and while they do not by themselves cause PD in ageing WT mice, they may become lethal for DA neurons weakened by PLA2g6 deficiency, sustained $\mathrm{Ca}^{2+}$ store depletion and autophagic dysfunction. It is appealing to speculate that idiopathic or genetic loss of PLA2g6-dependent SOCE function could be especially detrimental to DA neurons, which are physiologically weakened by excessive mitochondrial oxidant stress due to the life-long pacemaker activity of $\mathrm{Ca}_{\mathrm{V}} 1.3$ channels. Our study supports the idea of nigrostriatal degenerative processes as a complex phenomenon ${ }^{43,56,69}$ that goes beyond mitochondrial dysfunction, oxidative damage and/or 
defects in protein degradation. It is likely that impairment of PLA2g6-dependent store-operated $\mathrm{Ca}^{2+}$ signalling can initiate or in tandem with other age-related processes exacerbate a sequence of pathological events leading to demise of specific DA neurons in $\mathrm{SNc}$, and resulting in PD.

Altogether, our study presents empirical evidence for a previously unknown link between PARK14/PLA2g6, SOCE and human PD, and the data provide strong support for our conclusions. First, we found that primary skin fibroblasts from idPD patients have a characteristic cellular phenotype with pronounced deficiency in SOCE and significant autophagic dysfunction (Figs 1 and 7). Importantly, we demonstrated that such idPD phenotype (impaired SOCE and autophagic dysfunction) can be rescued by simple overexpression of the functional PLA2g6(L) (Fig. 7c,d). Second, the cells from fPD patient carrying PLA2g6 ${ }^{\mathrm{R}} 47 \mathrm{~W}$ mutation have the same cellular phenotype (loss of PLA2g6 activation by the stores, deficient SOCE, depleted stores and autophagic dysfunction) as we found in idPD patients (Figs 1 and 7). Third, human PD-associated mutations in PARK14 (F72L and R747W) appeared to be sufficient to impair SOCE, deplete the stores and cause autophagic dysfunction (Fig. 6). Fourth, cellular phenotype of human idPD and PARK14 fPD patients can be mimicked by targeted impairment of PLA2g6 function in a new transgenic mouse model (Figs 1, 2 and 6). Importantly, impairment of SOCE and depletion of $\mathrm{Ca}^{2+}$ stores was found not only in fibroblasts but also in iPSCderived A9 midbrain DA neurons from PLA2g6 ex $2^{\mathrm{KO}}$ mice. Fifth, genetic impairment of $\mathrm{Ca}^{2+}$ signalling function of PLA2g6 in a new ex $2^{\mathrm{KO}}$ mouse model resulted in pronounced age-dependent PD-like phenotype that mimics idPD in humans.

The PLA2g6 ex $2^{\mathrm{KO}}$ mouse model exhibits not only PD-like motor dysfunction but also an anatomically selective depletion of nigral DA neurons resulting from an endogenous pan-neuronal deficit, and provides an important addition to existing mouse models of $\mathrm{PD}{ }^{55,56}$. PLA2g6 ex $2^{\mathrm{KO}}$ mouse reproduces several major parameters of human sporadic $\mathrm{PD}$, and combines autophagic dysfunction, progressive loss of DA neurons in $\mathrm{SNc}$ and age-dependent L-DOPA-sensitive PD-like motor dysfunction. This new mammalian model opens unique opportunities to investigate mechanisms contributing to the aetiology of sporadic PD, and provides a powerful tool for developing novel strategies for prevention and treatment of this devastating neurodegenerative disease.

\section{Methods}

Animals. A novel PLA2g6 ex $2^{\mathrm{KO}}$ transgenic mouse model, in which exon 2 of Pla2g6 gene was constitutively deleted, was custom created by GenOway (http://www.genoway.com/, France). The strategy for creation and results of model validation are presented in Supplementary Methods and Supplementary Figs 5-7. Heterozygote ex $2{ }^{\mathrm{KO}}$ males were backcrossed to C57BL/6 females for nine generations, and congenic B6.Cg-Pla2g $6^{\Delta E x 2-V B} / \mathrm{J}$ line was established. Owing to infertility of homozygous ex $2^{\mathrm{KO}}$ males, and the inability of homozygous females to sustain neonatal pups, cross-breeding of heterozygous mice was employed to obtain homozygous ex $2^{\mathrm{KO}}$ animals used in this study. Ageing male mice were used for in vivo studies, while female mice were used for preparation of MEFs. Experimental sets of homozygous ex $2 \mathrm{KO}$ and WT littermate males were housed and aged together. Animal number for each study group was determined for the experimental results to reach statistical significance with a power of $90 \%$ at $P<0.05$, or to demonstrate that there is no difference between the groups. Animals were maintained in an advanced pathogen-free facility with veterinary service and unlimited access to food and water. All experimental procedures were compliant with ethical regulations and approved by the Institutional Animal Care and Use Committee of Boston University.

Motor coordination tests. Ageing PLA2g6 ex $2^{\mathrm{KO}}$ and WT mice were monitored for the signs and severity of clinical symptoms, and motor deficit was initially assessed in arbitrary units (a.u.) using the following scale: $0=$ no abnormalities; $1=$ subtle signs of motor dysfunction; $2=$ clear signs of movement impairment, but sustained postural stability; 3 =impairment in movement and occasional postural instability; $4=$ strong ataxia and instability, but no difficulty with eating, drinking and grooming; $5=$ very strong ataxia resulting in difficulty with keeping sternal/upright position, and frequently falls when walking, but still able to eat, drink and groom, although with some difficulty.

Analysis of motor function was performed in age-matched $\mathrm{ex}_{2} \mathrm{KO}$ and WT animals using standard behaviour tests, as described in detail in Supplementary Methods. Balance beam test assessed the ability of ageing mice to maintain balance while walking along a narrow beam (the number of missteps per metre was counted). The pole test assessed the time that is needed for balance and orientation on the top of the pole. The rotarod test determined how long the mouse can maintain its balance and stay on a rotating rod. The grip test was used to objectively quantify the muscular strength of the forelimbs and hindlimbs. L-DOPA challenge test was performed on ex $2^{\mathrm{KO}}$ mice with motor deficits to determine the ability of L-DOPA to temporarily improve motor function, which was assessed using balance beam test. The data were summarized for each group as mean \pm s.e. The number of animals used for each study is identified on the graphs.

Brain slices: preparation, immunostaining and analysis. The brains were extracted following paraformaldehyde (4\%) perfusion, and cryopreserved in 15 and $30 \%(\mathrm{w} / \mathrm{v})$ sucrose solutions at $4{ }^{\circ} \mathrm{C}$. Brain sections were prepared using standard methods, as described in Supplementary Methods. Briefly, coronal 30- $\mu$ m-thick free-floating sections containing the SNc were collected using a staggering method, and sets of six brain sections were collated: each set contained similar sections from the rostral, middle and caudal parts of the SNc region. Investigators were blinded during sectioning, $\mathrm{TH}$ staining, unbiased stereology and analysis of PAS staining, mouse phenotypes were revealed/confirmed only after data were generated. No samples were excluded from analysis in targeted age groups.

Blinded unbiased stereological analysis of DA neurons in SNc of age-matched pairs of ex $2^{\mathrm{KO}}$ and WT littermates was performed using standard methodology described in Supplementary Methods. Briefly, the matching sets of brain slices were stained with TH rabbit polyclonal antibody (Calbiochem) and $3,3^{\prime}$-diaminobenzidine horseradish peroxidase (HRP) substrate (Vector Laboratories). To estimate the number of $\mathrm{TH}+$ neurons, matching sets of six sections from SNc area of the brain of each experimental animal were analysed using a Nikon Eclipse E600 microscope and the Stereo-Investigator v11.01.2 software. The total number of $\mathrm{TH}+$ cells in the SNc was estimated using the optical fractionator technique, as described in Supplementary Methods. The data were summarized for each group as average \pm s.e. Paired $t$-test was used for statistical analysis of the differences within the matching pairs of $\mathrm{WT}$ and $\mathrm{ex} 2{ }^{\mathrm{KO}}$ littermates. The numbers of littermate pairs used for these studies is identified on the graphs.

PAS staining and analysis was performed using Sigma kit (\#395B) and standard procedures, as described in Supplementary Methods. PAS-positive cells (stained rose to magenta with blue to black nuclei) were counted in $\mathrm{SNc}$, hippocampus and M1/M2 motor cortex regions. Summary data show average number of PAS + neurons per $\mathrm{mm}^{2}$ ( \pm s.e.) from three pairs of age-matched WT and ex2 $2^{\mathrm{KO}} 16$-month-old animals.

Immunostaining for $\mathrm{TH}$ and LC3 was done using primary chicken polyclonal anti-TH antibody (Abcam ab76442), primary rabbit polyclonal anti-LC3 antibody (Cell Signaling \#2775), secondary goat anti-rabbit Alexa488 (Invitrogen A11034) and goat anti-chicken Alexa594 (Abcam AB150172) antibodies, as described in Supplementary Methods.

Nissl (cresyl violet) staining was performed using a standard procedure described in Supplementary Methods.

Primary MEFs. MEFs were isolated from WT, ex $2^{\mathrm{KO}}$ and Orai1 ${ }^{\mathrm{KO}}$ embryos (14.5 days old). $\mathrm{Ex} 2^{\mathrm{KO}}$ embryos were obtained from homozygote ex $2^{\mathrm{KO}}$ females mated with heterozygote ex $2^{\mathrm{KO}}$ males. Orai $1^{\mathrm{KO}}$ embryos were obtained by the cross-breeding of heterozygous Orail ${ }^{\mathrm{KO}}$ mice. Each embryo was genotyped. Head, vertebral column, dorsal root ganglia and all internal organs were removed and discarded; the remaining embryonic tissue was manually dissociated and incubated in $0.25 \%$ trypsin (Gibco) for 15-30 min. Cells from each embryo were plated onto a $10-\mathrm{cm}$ tissue culture dish in MEF media (Dulbecco's modified Eagle medium (DMEM); Mediatech Inc.) containing 10\% fetal bovine serum (FBS; Hyclone), non-essential amino acids, sodium pyruvate and penicillin/streptomycin (Invitrogen). After reaching confluence, primary MEFs from WT and $\mathrm{ex}_{2} \mathrm{KO}$ embryos were tested (and confirmed to be negative) for mycoplasma contamination, collected and stored in liquid nitrogen for future use. Only MEFs from passages 2-3 were used for experiments, and studied after $24-48 \mathrm{~h}$ in culture. For each experimental condition, independent experiments were performed on the cells from three different MEF preparations; the number of cells (or samples) for each condition is shown in the figures, and/or stated in figure legends.

Transfection of MEFs was performed using the Amaxa Nucleofector system (Lonza, Allendale, NJ, USA). Briefly, the cells were plated in a six-well plate at a density of 200,000 per well. After $24 \mathrm{~h}$ the cells from each well were collected, centrifuged, resuspended in $100 \mu$ lof electroporation solution (Mirus Bio, Madison, WI, USA), mixed with $2 \mu \mathrm{g}$ of the recombinant plasmid DNA and transfected using the T020 programme. After electroporation, the cells were plated in $2 \mathrm{ml}$ of warm DMEM containing 10\% FBS and $1 \%$ penicillin/streptomycin, and grown for $24-48 \mathrm{~h}$, as specified. Transfection efficiency (verified by expression of GFP or LC $3^{\text {mCherry-eGFP) }}$ was $>70 \%$. 
iPSC-derived A9 midbrain DA neurons. Derivation of iPSC from MEFs was performed using STEMCCA approach (see Supplementary Methods for details). Colonies positive for alkaline phosphatase, Oct3/4, Zfp96, Nanog and ERas were expanded and banked for neural differentiation. The cultures were routinely checked and confirmed to be negative for mycoplasma.

Differentiation of iPSC into DA neurons was done using standard protocol ${ }^{42}$ by first inducing formation of embryoid bodies in non-adherent conditions for 4 days in KSR media. Cells were then transferred to adherent plates and incubated in ITS media (DMEM/F12 (Gibco) $+1 \times$ ITS Supplement (Sigma I13146) $+1 \mu \mathrm{g} \mathrm{ml}^{-1}$ bovine fibronectin (Sigma F1141)) for 6-10 days to induce ectoderm formation. Cells were then expanded onto polyornithine-/fibronectin-coated coverslips in media containing N2 Max supplement, FGF2, FGF-8b, Shh-N and ascorbic acid for 5-7 days until cells reach confluency. Neural identity was confirmed by staining against $\beta I I I$ tubulin and nestin. Final differentiation into DA neurons was done by incubating the cells for 10 more days in minimal media (DMEM/F12 (Gibco) $+1 \times \mathrm{N} 2 \mathrm{Max}+200 \mu \mathrm{M}$ ascorbic acid (Sigma A4403)). Confirmation of DA neuron identity was done by staining the cells for TH (Abcam ab76442), Dopamine transporter (Abcam ab5990) and vesicular monoamine transporter 2 (Abcam 70808).

Human primary skin fibroblasts. The samples of hPSF (see Supplementary Table 1 for all details) were obtained from the NINDS Cell Line Repository

(http://ccr.coriell.org/ninds). Each sample was verified to be mycoplasma-free by PCR. The hPSFs from individual donors were cultured as recommended. Briefly, hPSF cells were grown in Eagle's minimum essential medium (Gibco) supplemented with 15\% FBS (Atlanta Biologicals, GA, USA), $2 \mathrm{mM}$ L-glutamine (Gibco) and 1\% penicillin/ streptomycin (Gibco), and passaged 1:4 every 7 days. Only hPSF from early passages (3-5) were used in these studies. For each experimental condition, at least three independent experiments were performed; the numbers of cells (or samples) for each condition is shown in the figures, and/or stated in figure legends.

Transfection of hPSFs was performed using the Amaxa Nucleofector system. The cells were plated at a density of 300,000 per well in a six-well plate. After $24 \mathrm{~h}$ the cells from individual wells were collected, centrifuged, resuspended in $100 \mu \mathrm{l}$ of electroporation solution, mixed with $2 \mu \mathrm{g}$ of total plasmid DNA and transfected using the U023 programme. After electroporation, the cells were plated in fibronectin-coated $\left(2.5 \mu \mathrm{g} \mathrm{cm}^{-2}\right.$, Sigma, St Louis, MO, USA) $35-\mathrm{mm}$ glass-bottom dishes. Live-cell imaging was performed $48 \mathrm{~h}$ after transfection. Transfection efficiency (verified by expression of GFP or LC3 $3^{\text {mCherry-eGFP }}$ ) was about $50 \%$.

$\mathrm{Ca}^{2+}$ imaging. Intracellular $\mathrm{Ca}^{2+}$ studies were performed using standard Fura-2 imaging technique (for details see Supplementary Methods). Briefly, the cells were loaded with Fura-2/AM $\left(5 \mu \mathrm{M}\right.$; Invitrogen) and cytosolic $\mathrm{Ca}^{2+}$ (using $F_{340} / F_{380}$ ratio) was recorded simultaneously in individual cells. Representative traces in the figures show average ( \pm s.d.) responses from up to 20 cells recorded simultaneously. $\mathrm{Ca}^{2+}$ changes are shown by $\Delta$ Ratio $\left(\Delta F_{340} / F_{380}\right)$, which is the difference between the basal and peak values of $\mathrm{Ca}^{2+}$ responses. Summary bar graphs show the average $( \pm$ s.e. $)$ from three independent experiments for each condition; the total number of cells in each experimental group is identified on the graphs.

For SOCE recording, the cells were placed in $\mathrm{Ca}^{2+}$-free extracellular solution $(130 \mathrm{mM} \mathrm{NaCl}, 4.6 \mathrm{mM} \mathrm{KCl}, 2 \mathrm{mM} \mathrm{MgCl} 2,10 \mathrm{mM}$ HEPES/Na, $5 \mathrm{mM}$ glucose, $100 \mu \mathrm{M}$ EGTA, pH 7.4), and acute (5-20 min) application of $5 \mu \mathrm{M}$ TG (Sigma) was used to irreversibly inhibit SERCA activity, and allow $\mathrm{Ca}^{2+}$ to leak out from the stores, thus causing ER store depletion. Acute treatment with TG did not cause unfolded protein response (Supplementary Fig. 4). SOCE was measured in response to extracellular application of $2-2.5 \mathrm{mM} \mathrm{Ca}^{2+}$ in the presence of TG. Concentration and time of acute TG treatment was titrated for each cell type to ensure $>90 \%$ loss of $\mathrm{Ca}^{2+}$ from TG-sensitive stores at the time of $\mathrm{Ca}^{2+}$ addition. As an alternative to TG, in some experiments SOCE was evoked by acute 5 -min treatment with $400 \mu \mathrm{M}$ TPEN $\left(N, N, N^{\prime}, N^{\prime}\right.$-Tetrakis(2-pyridylmethyl) ethylenediamine, Sigma, USA). To inhibit PLA2g6 activity, (S)-BEL (S-bromoenol lactone, Cayman, USA) was applied (10$50 \mu \mathrm{M}$ for $20 \mathrm{~min}$ in serum-free medium at $37^{\circ} \mathrm{C}$ ) to the cells after their loading with Fura-2/AM, and was washed away before the start of experiment.

Release of $\mathrm{Ca}^{2+}$ from intracellular stores was measured in $\mathrm{Ca}^{2+}$-free extracellular solution in response to acute application of ionomycin (Sigma) at concentration enough to release $>90 \%$ of $\mathrm{Ca}^{2}+$ from TG-sensitive stores (titrated for each cell type): $1 \mu \mathrm{M}$ for MEFs, $0.1 \mu \mathrm{M}$ for hPSF and $0.1 \mu \mathrm{M}$ for iPSC-derived DA neurons.

Fura-2 recordings in MEFs were done at $20-22^{\circ} \mathrm{C}$ using dual-excitation Intracellular Imaging system (Intracellular Imaging Inc., Cincinnati, $\mathrm{OH}$ ), unless specified differently. Fura-2 fluorescence in hPSF and iPSC-derived DA neurons was recorded using Nikon Eclipse Ti (Nikon, Melville, NY)/Lambda DG-4 system (Sutter Instrument, Novato, CA) equipped with Fura-2 cube (FURA2-C-NTEZERO, Semrock, Rochester, NY), CFI S Plan Fluor $\times 20 / 0.45$ objective (Nikon), perfect focus, $\mathrm{X} / \mathrm{Y}$ positioning and multiple field stitching. Fura-2 recordings in iPSC-derived neurons were done at $37^{\circ} \mathrm{C}$ and the data from the whole field, which were labelled with a scratch for later identification, were stored. The cells were then immediately fixed, stained for TH and imaged. The paired images (Fura-2 recordings and ICC stainings) were superimposed and $\mathrm{Ca}^{2+}$ responses were analysed in $\mathrm{TH}+\mathrm{iPSC}$-derived A9 midbrain DA neurons.

For comparison of $\mathrm{Ca}^{2+}$ responses in iPSC-derived DA neurons and MEFs, a separate set of experiments was performed with both cell types treated and studied at exactly the same experimental conditions $\left(37^{\circ} \mathrm{C}\right)$ using the same settings on Nikon Eclipse Ti/Lambda DG-4 system.

Live-cell imaging and co-localization analysis. Live-cell imaging was done using Nikon Ti inverted fluorescence microscope equipped with a Perfect Focus system and environmental chamber (InVivo Scientific). Live cells transfected with LC $3^{\text {mCherry-eGFP }}$ (alone, or in combination with PLA2g6, or its mutants) were imaged in glass-bottom dishes in culture medium at $37^{\circ} \mathrm{C}$ and $5 \% \mathrm{CO}_{2}$. Images of individual cells were taken using a $\times 60 / 1.4$ Plan-Apochromat oil immersion objective (Nikon) and filter sets for GFP (excitation: 465-496; emission: 515-555) and Texas Red (excitation: 540-580; emission: 600-660). Images of representative cells were analysed using ImageJ (Wayne Rasband, NIH) software. For increased accuracy and better visualization, the background was subtracted (rolling ball radius: $5.35 \mu \mathrm{m}$ ), and the unsharp mask filter (radius: $0.428 \mu \mathrm{m}$; mask weight: 0.6 ) was identically applied to both green and red channels for all images. Analysis of mCherry and eGFP co-localization was done using Pearson's correlation coefficient ${ }^{70}$. Briefly, the normalized covariance image of each cell was computed using ImageJ and the equation:

$$
g_{i j}=\frac{e_{i j} f_{i j}-\bar{E} \bar{F}}{\sqrt{\left[\overline{E^{2}}-(\bar{E})^{2}\right]\left[\overline{F^{2}}-(\bar{F})^{2}\right]}}
$$

where $e_{i j}$ and $f_{i j}$ are the pixels in the respective panels, $\bar{E}$ and $\bar{F}$ are the mean values of the pixels and $\overline{E^{2}}$ and $\overline{F^{2}}$ are the means of the squared pixel values. The average of the values of all pixels $g_{i j}$ was equal to Pearson's correlation coefficient. A correlation map was generated using the Interactive $3 \mathrm{D}$ surface plot plugin in ImageJ, and the positive contribution at each pixel to the correlation coefficient is displayed. Summary data show the average \pm s.e. of correlation coefficients and sizes of fluorescent particles in a total of 15 cells from three independent experiments (five representative cells per experiment).

Confocal and TIRF imaging. Confocal imaging of brain slices was done using LSM710 Duo imaging system (Zeiss, Thornwood, NY,USA) with either a $\times 20$ non-immersion or $\times 63 / 1.4$ Plan-Apochromat oil immersion objectives, as described in Supplementary Methods. Total internal reflection fluorescence (TIRF) imaging of the bottom plane of MEFs was done using a Nikon Ti inverted fluorescence microscope with a $\times 60 / 1.49$ Apo-TIRF oil objective (Nikon) and a filter set for GFP illumination.

PLA2g6 activity. A modified PLA2 assay kit (Cayman for MEFs and AbCam for hPSF) was used as previously described ${ }^{21,26}$. Briefly, each sample of live hPSF or MEF cells was homogenized using a cold lysis buffer ( $10 \mathrm{mM}$ Tris- $\mathrm{HCl}, \mathrm{pH} 7.0$, $300 \mathrm{mM}$ sucrose, $0.5 \%$ Triton X-100). To identify specific activity of $\mathrm{Ca}^{2+}$. independent PLA2g6, the assay buffers were modified to contain no $\mathrm{Ca}^{2+}$. To assess catalytic activity of PLA2g6, the cells were homogenized and treated with $10 \mathrm{mM}$ EGTA for $10 \mathrm{~min}$, which is known to directly displace inhibitory calmodulin and fully activate PLA2g6. For the analysis of PLA2g6 activation by store depletion, live cells were pretreated with TG $(5 \mu \mathrm{M}$ for $10 \mathrm{~min})$ before homogenization, and homogenates were not treated with $10 \mathrm{mM}$ EGTA. The specificity of PLA2g6 activity in both cases was confirmed by its inhibition with S-BEL $(25 \mu \mathrm{M}$ for $10 \mathrm{~min})$, a chiral-specific suicidal substrate ${ }^{38}$ that discriminates PLA2g6 from all other phospholipases. PLA2g6 activity in each sample was assayed (in triplicates) by incubating the samples with the substrate, 1-hexadecyl-2arachidonoylthio-2-deoxy-sn-glycero-3-phosphorylcholine for $1 \mathrm{~h}$ at room temperature in a modified $\mathrm{Ca}^{2}+$-free assay buffer $(10 \mathrm{mM}$ HEPES, $\mathrm{pH} 7.4$, $300 \mathrm{mM} \mathrm{NaCl}, 60 \%$ glycerol, $8 \mathrm{mM}$ Triton X-100, $4 \mathrm{mM}$ EGTA and $2 \mathrm{mg} \mathrm{ml}^{-1}$ bovine serum albumin). The reaction was stopped and the generation of free thiols was visualized by addition of DTNB (5,5'-dithiobis-(2-nitrobenzoic acid)) for $5 \mathrm{~min}$ : the absorbance was determined at $405 \mathrm{~nm}$ using a standard microplate reader. In calculations of specific PLA2g6 activity a value of $10 \mathrm{mM}^{-1}$ was used as extinction coefficient for DTNB at $405 \mathrm{~nm}$.

The activity of PLA2g6 was expressed in $\mathrm{nM} \mathrm{min}{ }^{-1}$ per $\mathrm{mg}$ of protein.

Western blot. Tissue preparation and western blot analysis was done using standard approaches (see Supplementary Methods for details).

Antibodies. The following primary antibodies were used in this study: rabbit polyclonal anti-TH antibody (Calbiochem, \#657012); chicken polyclonal anti-TH antibody (Abcam, ab76442); rabbit polyclonal anti-LC3B antibody (Cell Signaling, \#2775); rabbit polyclonal anti-vesicular monoamine transporter 2 antibody (Abcam, ab70808); rat monoclonal anti-dopamine transporter antibody (Abcam, ab5990); rabbit polyclonal anti-PIN antibody targeting mouse PLA2g6 PIN domain (encoded by exon 8b, which is present in (L), but spliced out in (S) variant) were custom made by Yenzym Antibodies, LLC (San Francisco, CA), rabbit polyclonal anti-LC3B antibody (MBL, PD014); and monoclonal anti- $\beta$-actin antibody (Sigma, A1978). Secondary antibodies are the following: goat anti-rabbit Alexa488 (Invitrogen A11034), goat anti-chicken Alexa594 (Abcam ab150172) and goat anti-rat Alexa647 (Abcam ab150167) were used for imaging; HRP-conjugated 
anti-rabbit (Dako, K4002) was used for 3,3'-diaminobenzidine staining; HRP-conjugated anti-mouse (Cell Signaling, \#7076) or anti-rabbit IgG (Cell Signaling, 7074) was used as secondary Ab for western blots.

Quantitative reverse transcription-PCR. Total RNA was isolated from hPSF of each individual donor and from primary MEFs from ex $2{ }^{\mathrm{KO}}$ and WT mice using High Pure RNA isolation kit (Roche Applied Science). Concentration and quality of samples were confirmed spectrophotometrically. RNA was reverse transcribed using High Capacity RNA-to-cDNA Kit (Life Technologies), and cDNA (equivalent of $200 \mathrm{ng}$ RNA) was analysed per each reaction (in duplicates for technical control) in quantitative PCR on StepOnePlus Real Time PCR System (Applied Biosystems). For the full list of TaqMan gene expression assays, see Supplementary Methods. The relative expression level for each gene was normalized to the level of GAPDH in the same sample.

DNA constructs. Tandem LC $3^{\text {mCherry-cGFP }}$ construct was obtained from Addgene. cDNA for human PLA2g6(L) variant ${ }^{39}$ (Genbank \#AF064594) was a kind gift from Dr Brian P. Kennedy (Karolinska Institute, Stockholm, Sweden) and MERCK FROSST CANADA Inc. His-tagged and/or myc-tagged expression constructs of PLA2g6(L) were created by PCR subcloning of the full-length long variant of human PLA2g6(L) into pcDNA3.1 plasmid (see Supplementary Methods for details). F72L and A80T mutants of myc PLA2g6(L ${ }^{\text {his }}$ were created by Mutagenex (USA), and confirmed by sequencing.

Statistical analysis. A two-sided unpaired $t$-test was used for comparison among different data sets, unless stated differently. Normal distribution was confirmed using D'Agostino-Pearson omnibus normality test $(\alpha<0.05)$. A two-sided paired $t$-test was used for stereological analysis. One-way analysis of variance was used for the analysis of the data from L-DOPA challenge test. The difference between data sets was considered significant at $P<0.05 ; P$ values are identified in the figures and legends as ${ }^{\star} P<0.05,{ }^{* *} P<0.01,{ }^{* * *} P<0.001$. Summary data represent average \pm s.e. or s.d., as specified in the figure legends.

\section{References}

1. Trinh, J. \& Farrer, M. Advances in the genetics of Parkinson disease. Nat. Rev. Neurol. 9, 445-454 (2013).

2. Antony, P. M. A., Diederich, N. J., Kruger, R. \& Balling, R. The hallmarks of Parkinson's disease. FEBS J. 280, 5981-5993 (2013).

3. Dexter, D. T. \& Jenner, P. Parkinson disease: from pathology to molecular disease mechanisms. Free Radic. Biol. Med. 62, 132-144 (2013).

4. Obeso, J. A. et al. Missing pieces in the Parkinson's disease puzzle. Nat. Med. 16, 653-661 (2010)

5. Paisan-Ruiz, C. et al. Characterization of PLA2G6 as a locus for dystoniaparkinsonism. Ann. Neurol. 65, 19-23 (2009).

6. Yoshino, H. et al. Phenotypic spectrum of patients with PLA2G6 mutation and PARK14-linked parkinsonism. Neurology 75, 1356-1361 (2010).

7. Paisan-Ruiz, C. et al. Widespread Lewy body and tau accumulation in childhood and adult onset dystonia-parkinsonism cases with PLA2G6 mutations. Neurobiol. Aging 33, 814-823 (2010).

8. Tomiyama, H. et al. PLA2G6 variant in Parkinson's disease. J. Hum. Genet. 56, 401-403 (2011).

9. Kauther, K. M., Hoft, C., Rissling, I., Oertel, W. H. \& Moller, J. C. The PLA2G6 gene in early-onset Parkinson's disease. Mov. Disord. 26, 2415-2417 (2011).

10. Sina, F., Shojaee, S., Elahi, E. \& Paisan-Ruiz, C. R632W mutation in PLA2G6 segregates with dystonia-parkinsonism in a consanguineous Iranian family. Eur. J. Neurol. 16, 101-104 (2009).

11. Lu, C. S. et al. PLA2G6 mutations in PARK14-linked young-onset parkinsonism and sporadic Parkinson's disease. Am. J. Med. Genet. 159B, 183-191 (2012)

12. Dennis, E. A., Cao, J., Hsu, Y. H., Magrioti, V. \& Kokotos, G. Phospholipase A2 enzymes: physical structure, biological function, disease implication, chemical inhibition, and therapeutic intervention. Chem. Rev. 111, 6130-6185 (2011).

13. Bao, S. et al. Male mice that do not express group VIA phospholipase A2 produce spermatozoa with impaired motility and have greatly reduced fertility. J. Biol. Chem. 279, 38194-38200 (2004).

14. Malik, I. et al. Disrupted membrane homeostasis and accumulation of ubiquitinated proteins in a mouse model of infantile neuroaxonal dystrophy caused by PLA2G6 mutations. Am. J. Pathol. 172, 406-416 (2008).

15. Shinzawa, K. et al. Neuroaxonal dystrophy caused by group VIA phospholipase A2 deficiency in mice: a model of human neurodegenerative disease. $J$. Neurosci. 28, 2212-2220 (2008).

16. Wada, H. et al. Establishment of an improved mouse model for infantile neuroaxonal dystrophy that shows early disease onset and bears a point mutation in Pla2g6. Am. J. Pathol. 175, 2257-2263 (2009).

17. Beck, G. et al. Neuroaxonal dystrophy in calcium-independent phospholipase A2beta deficiency results from insufficient remodeling and degeneration of mitochondrial and presynaptic membranes. J. Neurosci. 31, 11411-11420 (2011).

18. Zhao, Z. et al. Genetic ablation of PLA2G6 in mice leads to cerebellar atrophy characterized by purkinje cell loss and glial cell activation. PLOS ONE 6, e26991 (2011).

19. Strokin, M., Seburn, K. L., Cox, G. A., Martens, K. A. \& Reiser, G. Severe disturbance in the $\mathrm{Ca} 2+$ signaling in astrocytes from mouse models of human infantile neuroaxonal dystrophy with mutated Pla2g6. Hum. Mol. Genet. 21, 2807-2814 (2012).

20. Engel, L. A., Jing, Z., O’Brien, D. E., Sun, M. \& Kotzbauer, P. T. Catalytic function of PLA2G6 is impaired by mutations associated with infantile neuroaxonal dystrophy but not dystonia-parkinsonism. PLoS ONE 5, e12897 (2010).

21. Smani, T. et al. A novel mechanism for the store-operated calcium influx pathway. Nat. Cell Biol. 6, 113-120 (2004).

22. Bolotina, V. M. Orai, STIM1 and iPLA2beta: a view from a different perspective. J. Physiol. (Lond.) 586, 3035-3042 (2008).

23. Bolotina, V. M. in Store-Operated Calcium Entry (SOCE) Pathways (ed. Groschner, K.) 115-130 (Springer, 2012).

24. Gwozdz, T., Dutko-Gwozdz, J., Schafer, C. \& Bolotina, V. M. Overexpression of Orail and STIM1 proteins alters regulation of store-operated Ca2 + entry by endogenous mediators. J. Biol. Chem. 287, 22865-22872 (2012).

25. Gwozdz, T., Dutko-Gwozdz, J., Zarayskiy, V., Peter, K. \& Bolotina, V. M. How strict is the correlation between STIM1 and Orail expression, puncta formation, and ICRAC activation? Am. J. Physiol. Cell Physiol. 295, C1133-C1140 (2008).

26. Csutora, P. et al. Activation mechanism for CRAC current and store-operated $\mathrm{Ca}^{2+}$ entry: calcium influx factor and $\mathrm{Ca}^{2+}$-independent phospholipase A 2 b-mediated pathway. J. Biol. Chem. 281, 34926-34935 (2006).

27. Martinez, J. \& Moreno, J. J. Role of Ca2 + -independent phospholipase A2 and cytochrome P-450 in store-operated calcium entry in 3T6 fibroblasts. Biochem. Pharmacol. 70, 733-739 (2005).

28. Singaravelu, K., Lohr, C. \& Deitmer, J. W. Regulation of store-operated calcium entry by calcium-independent phospholipase A2 in rat cerebellar astrocytes. J. Neurosci. 26, 9579-9592 (2006).

29. Boittin, F. X. et al. Ca2 +-independent phospholipase A2 enhances storeoperated $\mathrm{Ca} 2+$ entry in dystrophic skeletal muscle fibers. J. Cell Sci. 119, 3733-3742 (2006).

30. Boittin, F. X., Gribi, F., Serir, K. \& Beny, J. L. Ca2 + -independent PLA2 controls endothelial store-operated $\mathrm{Ca} 2+$ entry and vascular tone in intact aorta. Am. J. Physiol. Heart Circ. Physiol. 295, H2466-H2474 (2008).

31. Ross, K., Whitaker, M. \& Reynolds, N. J. Agonist-induced calcium entry correlates with STIM1 translocation. J. Cell. Physiol. 211, 569-576 (2007).

32. Vig, M. et al. CRACM1 is a plasma membrane protein essential for storeoperated Ca2 + entry. Science 312, 1220-1223 (2006).

33. Putney, J. W. The physiological function of store-operated calcium entry. Neurochem. Res. 36, 1157-1165 (2011).

34. Parekh, A. B. Store-operated CRAC channels: function in health and disease. Nat. Rev. Drug Discov. 9, 399-410 (2010).

35. Lewis, R. S. Store-operated calcium channels: new perspectives on mechanism and function. Cold Spring Harb. Perspect. Biol. 3, 1-24 (2011).

36. Zarayskiy, V. et al. Store-operated Orail and IP3 receptor-operated TRPC1 channel. Channels (Austin.) 1, 246-252 (2007).

37. Zakharov, S. I. et al. Diethylstilbestrol is a potent inhibitor of store-operated channels and capacitative Ca2 + influx. Mol. Pharmacol. 66, 702-707 (2004).

38. Jenkins, C. M., Han, X., Mancuso, D. J. \& Gross, R. W. Identification of calcium-independent phospholipase A2 (iPLA2) beta, and Not iPLA2gamma, as the mediator of arginine vasopressin-induced arachidonic acid release in A-10 smooth muscle cells. Enantioselective mechanism-based discrimination of mammalian iPLA2s. J. Biol. Chem. 277, 32807-32814 (2002).

39. Larsson, P. K., Claesson, H. E. \& Kennedy, B. P. Multiple splice variants of the human calcium-independent phospholipase A2 and their effect on enzyme activity. J. Biol. Chem. 273, 207-214 (1998).

40. Hofer, A. M., Fasolato, C. \& Pozzan, T. Capacitative $\mathrm{Ca}^{2+}$ entry is closely linked to the filling state of internal $\mathrm{Ca}^{2}+$ stores: a study using simultaneous measurements of $\mathrm{I}_{\text {crac }}$ and intraluminal $\left[\mathrm{Ca}^{2+}\right]$. J. Cell Biol. 140, 325-334 (1998).

41. Csutora, P. et al. Novel role for STIM1 as a trigger for calcium influx factor production. J. Biol. Chem. 283, 14524-14531 (2008).

42. Lee, S. H., Lumelsky, N., Studer, L., Auerbach, J. M. \& McKay, R. D. Efficient generation of midbrain and hindbrain neurons from mouse embryonic stem cells. Nat. Biotechnol. 18, 675-679 (2000).

43. Surmeier, D. J., Guzman, J. N., Sanchez, J. \& Schumacker, P. T. Physiological phenotype and vulnerability in Parkinson's disease. Cold Spring Harb. Perspect. Med. 2, a009290 (2012).

44. Collier, T. J., Kanaan, N. M. \& Kordower, J. H. Ageing as a primary risk factor for Parkinson's disease: evidence from studies of non-human primates. Nat. Rev. Neurosci. 12, 359-366 (2011). 
45. Albin, R. L., Young, A. B. \& Penney, J. B. The functional anatomy of disorders of the basal ganglia. Trends Neurosci. 18, 63-64 (1995).

46. Savitt, J. M., Dawson, V. L. \& Dawson, T. M. Diagnosis and treatment of Parkinson disease: molecules to medicine. J. Clin. Invest. 116, 1744-1754 (2006).

47. Lynch-Day, M. A., Mao, K., Wang, K., Zhao, M. \& Klionsky, D. J. The role of autophagy in Parkinson's disease. Cold Spring Harb. Perspect. Med. 2, a009357 (2012).

48. Dehay, B. et al. Lysosomal impairment in Parkinson's disease. Mov. Disord. 28, 725-732 (2013).

49. Orenstein, S. J. et al. Interplay of LRRK2 with chaperone-mediated autophagy. Nat. Neurosci. 16, 394-406 (2013).

50. Cuervo, A. M., Stefanis, L., Fredenburg, R., Lansbury, P. T. \& Sulzer, D. Impaired degradation of mutant + i-synuclein by chaperone-mediated autophagy. Science 305, 1292-1295 (2004).

51. Tong, Y. et al. Loss of leucine-rich repeat kinase 2 causes impairment of protein degradation pathways, accumulation of alpha-synuclein, and apoptotic cell death in aged mice. Proc. Natl Acad. Sci. USA 107, 9879-9884 (2010).

52. Klionsky, D. J. et al. Guidelines for the use and interpretation of assays for monitoring autophagy. Autophagy 8, 445-544 (2012).

53. Ganley, I., Wong, P. M., Gammoh, N. \& Jiang, X. Distinct autophagosomallysosomal fusion mechanism revealed by thapsigargin-induced autophagy arrest. Mol. Cell 42, 731-743 (2011).

54. Vig, M. et al. Defective mast cell effector functions in mice lacking the CRACM1 pore subunit of store-operated calcium release-activated calcium channels. Nat. Immunol. 9, 89-96 (2008).

55. Lee, Y., Dawson, V. L. \& Dawson, T. M. Animal models of Parkinson's disease: vertebrate genetics. Cold Spring Harb. Perspect. Med. 2, 1-13 (2012).

56. Chesselet, M. F. \& Richter, F. Modelling of Parkinson's disease in mice. Lancet Neurol. 10, 1108-1118 (2011).

57. Berridge, M. J., Bootman, M. D. \& Roderick, H. L. Calcium signalling: dynamics, homeostasis and remodelling. Nat. Rev. Mol. Cell Biol. 4, 517-529 (2003).

58. Goldberg, J. A. et al. Calcium entry induces mitochondrial oxidant stress in vagal neurons at risk in Parkinson's disease. Nat. Neurosci. 15, 1414-1421 (2012).

59. Surmeier, D. J., Guzman, J. N., Sanchez-Padilla, J. \& Schumacker, P. T. The role of calcium and mitochondrial oxidant stress in the loss of substantia nigra pars compacta dopaminergic neurons in Parkinson's disease. Neuroscience 198, 221-231 (2011).

60. Selvaraj, S. et al. Neurotoxin-induced ER stress in mouse dopaminergic neurons involves downregulation of TRPC1 and inhibition of AKT/mTOR signaling. J. Clin. Invest. 122, 1354-1367 (2012).

61. Sun, S. et al. Reduced synaptic STIM2 expression and impaired store-operated calcium entry cause destabilization of mature spines in mutant presenilin mice. Neuron 82, 79-93 (2014).

62 . Wu, J. et al. Neuronal store-operated calcium entry pathway as a novel therapeutic target for Huntington's disease treatment. Chem. Biol. 18, 777-793 (2011).

63. Sammels, E., Parys, J. B., Missiaen, L., De Smedt, H. \& Bultynck, G. Intracellular $\mathrm{Ca} 2+$ storage in health and disease: a dynamic equilibrium. Cell Calcium 47, 297-314 (2010).

64. Mercado, G., Valdes, P. \& Hetz, C. An ERcentric view of Parkinson's disease. Trends Mol. Med. 19, 165-175 (2013).

65. Roussel, B. D. et al. Endoplasmic reticulum dysfunction in neurological disease. Lancet Neurol. 12, 105-118 (2013).

66. Hoozemans, J. J., van Haastert, E. S., Nijholt, D. A., Rozemuller, A. J. \& Scheper, W. Activation of the unfolded protein response is an early event in Alzheimer's and Parkinson's disease. Neurodegener. Dis. 10, 212-215 (2012).

67. Colla, E. et al. Endoplasmic reticulum stress is important for the manifestations of alpha-synucleinopathy in vivo. J. Neurosci. 32, 3306-3320 (2012).

68. Luk, K. C. et al. Pathological alpha-synuclein transmission initiates Parkinsonlike neurodegeneration in nontransgenic mice. Science 338, 949-953 (2012).

69. Dehay, B. \& Bezard, E. New animal models of Parkinson's disease. Mov. Disord. 26, 1198-1205 (2011).

70. Kirber, M. T., Chen, K. \& Keaney, Jr. J. F. YFP photoconversion revisited: confirmation of the CFP-like species. Nat. Methods 4, 767-768 (2007).

\section{Acknowledgements}

This study is dedicated to Mr Mark D. Bolotin, one of the victims of idPD. We are grateful to Dr G. Krapivinsky and Dr R. Cohen for valuable discussions, expert comments and edits to the manuscript. Special thanks to Dr R. Myers, Dr L. Goldstein and Dr P. Csutora for valuable discussions. We are thankful to S. Zaitsev, J. Meltzer, K. Ingraham, M.S. Khan, Y. Yao, M. Wojnarowicz and M. Segawa for technical assistance at different stages of this project, to G. Ngoh for help with primary MEF cells isolation, to Dr D.L. Rosene for expert guidance and help with stereological analysis and to Dr A. Sommer for help with iPSC. We acknowledge NINDS Cell Line Repository (http://ccr.coriell.org/ninds) for providing the samples of human skin fibroblasts. We also acknowledge help from the Center of Interdisciplinary Research, the Center for Regenerative Medicine (CReM), Cellular Imaging Core, and Instrumentation Core of the Department of Medicine at the Boston University School of Medicine. We are grateful for PLA2g6(L) cDNA provided by MERCK FROSST Canada Ltd and for Orail ${ }^{\mathrm{KO}}$ mice provided by Dr M. Vig. This work was partially supported by Research grant awards from the Michael J. FOX foundation, the US National Institutes of Health (RO1HL71793), Institut de Recherche Servier and the Department of Medicine at Boston University School of Medicine.

\section{Author contributions}

Q.Z. performed $\mathrm{Ca}^{2}+$ signalling studies in MEF cells, analysed PLA2g6 activity, conducted all western blot studies, performed gene expression studies in hPSF, performed motor coordination tests, monitored and genotyped ex $2^{\mathrm{KO}}$ mice colony, collected tissue samples, isolated, genotyped and cultured primary MEFs, prepared plasmids, analysed results and prepared figures; A.Y. performed all molecular studies of autophagic function in MEFs and primary human skin fibroblasts, created and characterized iPSC-derived DA neurons from WT and ex 2 KO mice, conducted $\mathrm{Ca}^{2+}$ signalling studies in human fibroblasts and mouse iPSC-derived DA neurons, performed gene expression studies, prepared brain slices, performed immunohistochemistry and high-resolution imaging/analysis, performed blinded stereological analysis of $\mathrm{TH}+$ neurons, cultured and transfected human and mouse fibroblasts, analysed results, prepared the figures for the manuscript, participated in writing and submission of the manuscript; G.R. created all expression constructs, conducted quantitative reverse transcription-PCR, verified ex2 deletion and participated in interpretation of some experiments; H.A. performed neuropathological analysis of mouse brain; C.T. performed immunostaining for LC3 in brain slices; N.A. performed preliminary analysis of ataxia in mice; M.K. made significant contributions to image analysis; G.M. provided guidance and significant help in creation and characterization of the iPSC-derived DA neurons; T.I. made significant contribution to mouse brain analysis, helped develop live animal tests for motor dysfunction and edited the manuscript; B.W. made significant contribution to the studies of autophagy, participated in data interpretation and PDrelated aspects of the study, and edited the manuscript; V.M.B. conceived, designed, organized, troubleshoot, supervised and coordinated all the studies, analysed the results, arranged the figures and wrote the manuscript; all authors agreed with its final version.

\section{Additional information}

Supplementary Information accompanies this paper at http://www.nature.com/ naturecommunications

Competing financial interests: The authors declare no competing financial interests.

Reprints and permission information is available online at http://npg.nature.com/ reprintsandpermissions/

How to cite this article: Zhou, Q. et al. Impairment of PARK14-dependent $\mathrm{Ca}^{2+}$ signalling is a novel determinant of Parkinson's disease. Nat. Commun. 7:10332 doi: $10.1038 /$ ncomms10332 (2016).

This work is licensed under a Creative Commons Attribution 4.0 International License. The images or other third party material in this article are included in the article's Creative Commons license, unless indicated otherwise in the credit line; if the material is not included under the Creative Commons license, users will need to obtain permission from the license holder to reproduce the material. To view a copy of this license, visit http://creativecommons.org/licenses/by/4.0/ 